\title{
An asymptotic analysis for a generalized Cahn-Hilliard system with fractional operators
}

\author{
Pierluigi Colli@, Gianni Gilardi and JUtrgen Sprekels
}

Abstract. In the recent paper "Well-posedness and regularity for a generalized fractional Cahn-Hilliard system" (Colli et al. in Atti Accad Naz Lincei Rend Lincei Mat Appl 30:437-478, 2019), the same authors have studied viscous and nonviscous Cahn-Hilliard systems of two operator equations in which nonlinearities of double-well type, like regular or logarithmic potentials, as well as nonsmooth potentials with indicator functions, were admitted. The operators appearing in the system equations are fractional powers $A^{2 r}$ and $B^{2 \sigma}$ (in the spectral sense) of general linear operators $A$ and $B$, which are densely defined, unbounded, selfadjoint, and monotone in the Hilbert space $L^{2}(\Omega)$, for some bounded and smooth domain $\Omega \subset \mathbb{R}^{3}$, and have compact resolvents. Existence, uniqueness, and regularity results have been proved in the quoted paper. Here, in the case of the viscous system, we analyze the asymptotic behavior of the solution as the parameter $\sigma$ appearing in the operator $B^{2 \sigma}$ decreasingly tends to zero. We prove convergence to a phase relaxation problem at the limit, and we also investigate this limiting problem, in which an additional term containing the projection of the phase variable on the kernel of $B$ appears.

\section{Introduction}

A research project that the three of us recently carried out in [15-17] deals with the well-posedness, regularity and optimal control for the abstract evolutionary system

$$
\begin{aligned}
& \partial_{t} \varphi+A^{2 r} \mu=0, \\
& \tau \partial_{t} \varphi+B^{2 \sigma} \varphi+F^{\prime}(\varphi)=\mu+f, \\
& \varphi(0)=\varphi_{0},
\end{aligned}
$$

where $A^{2 r}$ and $B^{2 \sigma}$, with $r>0$ and $\sigma>0$, denote fractional powers of the linear operators $A$ and $B$, respectively. These operators are supposed to be densely defined in $H:=L^{2}(\Omega)$, with $\Omega \subset \mathbb{R}^{3}$, selfadjoint and monotone, and to have compact resolvents. The above system is a generalization of the standard or viscous CahnHilliard system (depending on whether $\tau=0$ or $\tau>0$ ), which models a phase separation process taking place in the container $\Omega$. The particular sample case $A^{2 r}=$ $B^{2 \sigma}=-\Delta$ with homogeneous Neumann boundary conditions is included, indeed. The physical variables $\varphi$ and $\mu$ stand for the order parameter and the chemical

Mathematics Subject Classification: 35K45, 35K90, 35R11, 35B40

Keywords: Fractional operators, Cahn-Hilliard systems, Asymptotic analysis. 
potential, respectively, while $f$ is a given source term. Moreover, $F$ denotes a doublewell potential. We offer three physically significant examples for $F$, namely,

$$
\begin{aligned}
& F_{\text {reg }}(r):=\frac{1}{4}\left(r^{2}-1\right)^{2}, \quad r \in \mathbb{R}, \\
& F_{\log }(r):= \begin{cases}(1+r) \ln (1+r)+(1-r) \ln (1-r)-c_{1} r^{2}, & r \in(-1,1) \\
2 \ln (2)-c_{1}, & r \in\{-1,1\}, \\
+\infty, & r \notin[-1,1]\end{cases} \\
& F_{2 \text { obs }}(r):=-c_{2} r^{2} \text { if }|r| \leq 1 \text { and } F_{2 \text { obs }}(r):=+\infty \quad \text { if }|r|>1,
\end{aligned}
$$

where the constants $c_{i}$ in (1.5) and (1.6) satisfy $c_{1}>1$ and $c_{2}>0$ in order that all the three functions $F_{\text {reg }}, F_{\text {log }}, F_{2 \text { obs }}$ are nonconvex (they are just semiconvex, indeed). We point out that $F_{\text {reg }}, F_{\text {log }}, F_{2 \text { obs }}$ are called the classical regular potential, the logarithmic potential, and the double obstacle potential, respectively. In irregular situations like (1.6), one has to split $F$ into a nondifferentiable convex part $\widehat{\beta}$ (the indicator function of $[-1,1]$, in the case of (1.6)) and a smooth perturbation $\widehat{\pi}$. At the same time, one has to replace the derivative of the convex part by the subdifferential and to interpret (1.2) as a differential inclusion or, equivalently, as a variational inequality involving $\widehat{\beta}$ rather than its subdifferential, as actually done in [15].

Fractional versions of the Cahn-Hilliard system have been considered by different authors and are the subject of several papers. As for references regarding wellposedness and related problems, a rather large list of citations is given in [15]; we recall some concerned and recent literature also here, by mentioning $[1,2,8,21,30,33]$. Moreover, one can find a number of results regarding the asymptotic behavior of solutions, for the standard Cahn-Hilliard equations, for variants thereof, and for systems including the Cahn-Hilliard equations: without any claim of completeness, we can quote, e.g., [3,6,9-12,14,18-20,23-25,31,32,34,35]. These works mainly deal with the asymptotics with respect to parameters, or the study of the trajectories and related topics, or the existence of global or exponential attractors and their properties. A special role in our citations is played by the paper [13], where the longtime behavior of the solutions as well as an asymptotic analysis similar to the one we address here are investigated for a fractional system involving the Allen-Cahn equation.

In this paper, we consider the viscous case $\tau>0$ within the system (1.1)-(1.3) and study the asymptotic behavior of the solution as the parameter $\sigma$ involved in the operator $B^{2 \sigma}$ tends to zero. In this analysis, a crucial role is played by the orthogonal projection operator $P: H \rightarrow H$ onto the kernel ker $B$ of $B$. Indeed, if $\left(\varphi_{\sigma}, \mu_{\sigma}\right)$ denotes the solution to system (1.1)-(1.3) for an arbitrary $\sigma>0$, we prove that $\left(\varphi_{\sigma}, \mu_{\sigma}\right)$ converges as $\sigma \searrow 0$ to a solution $(\varphi, \mu)$ to the system

$$
\begin{aligned}
& \partial_{t} \varphi+A^{2 r} \mu=0, \\
& \tau \partial_{t} \varphi+\varphi-P \varphi+F^{\prime}(\varphi)=\mu+f, \\
& \varphi(0)=\varphi_{0} .
\end{aligned}
$$


In general, the convergence occurs along a subsequence, but in the case when the limit pair $(\varphi, \mu)$ uniquely solves (1.7)-(1.9), then the whole family $\left(\varphi_{\sigma}, \mu_{\sigma}\right)$ converges to $(\varphi, \mu)$ in the sense made precise by the statement of Theorem 2.5. Moreover, let us point out that the component $\varphi$ of the pair $(\varphi, \mu)$ is always uniquely determined, as it follows from the continuous dependence result given by Theorem 2.10. In the last part of the paper, we also discuss the limiting problem by proving a class of regularity results, quite interesting in our opinion, for which we have to use some sophisticated tools of interpolation theory. Our approach may be considered as an extension and further investigation with respect to the asymptotic results of [13, Section 7], in which a phase relaxation problem is obtained at the limit. Also in the present paper, Eq. (1.8) can be seen as an ordinary differential equation, but with a nonlocal structure due to the presence of the projection operator $P$. Our contribution here gives account of a new line of investigation that in our opinion should be further explored.

The rest of the paper is organized as follows: in the next Sect. 2, we list our assumptions and state our results. The corresponding proofs are given in the last two Sects. 3 and 4 .

\section{Statement of the problem and results}

In this section, we state precise assumptions and notations and present our results. Our framework is the same as in [15], and we briefly recall it here, for the reader's convenience. First of all, the open set $\Omega \subset \mathbb{R}^{3}$ is assumed to be bounded, connected and smooth. We use the notation

$$
H:=L^{2}(\Omega)
$$

and denote by $\|\cdot\|$ and $(\cdot, \cdot)$ the standard norm and inner product of $H$. As for the operators involved in our system, we postulate that

$$
A: D(A) \subset H \rightarrow H \text { and } B: D(B) \subset H \rightarrow H \text { are }
$$

unbounded, nonnegative, selfadjoint, linear operators with compact resolvents.

We denote by $\left\{\lambda_{j}\right\}$ and $\left\{\lambda_{j}^{\prime}\right\}$ the nondecreasing sequences of the eigenvalues of $A$ and $B$, and by $\left\{e_{j}\right\}$ and $\left\{e_{j}^{\prime}\right\}$ the corresponding (complete) systems of orthonormal eigenvectors, that is,

$$
\begin{aligned}
& A e_{j}=\lambda_{j} e_{j}, \quad B e_{j}^{\prime}=\lambda_{j}^{\prime} e_{j}^{\prime}, \quad \text { and }\left(e_{i}, e_{j}\right)=\left(e_{i}^{\prime}, e_{j}^{\prime}\right)=\delta_{i j} \text { for } i, j=1,2, \ldots, \\
& 0 \leq \lambda_{1} \leq \lambda_{2} \leq \ldots \text { and } 0 \leq \lambda_{1}^{\prime} \leq \lambda_{2}^{\prime} \leq \ldots, \text { with } \lim _{j \rightarrow \infty} \lambda_{j}=\lim _{j \rightarrow \infty} \lambda_{j}^{\prime}=+\infty
\end{aligned}
$$


where $\delta_{i j}$ denotes the Kronecker index. The power $A^{r}$ of $A$ with an arbitrary positive real exponent $r$ is given by

$$
\begin{aligned}
& A^{r} v=\sum_{j=1}^{\infty} \lambda_{j}^{r}\left(v, e_{j}\right) e_{j} \text { for } v \in V_{A}^{r}, \text { where } \\
& V_{A}^{r}:=D\left(A^{r}\right)=\left\{v \in H: \sum_{j=1}^{\infty}\left|\lambda_{j}^{r}\left(v, e_{j}\right)\right|^{2}<+\infty\right\} .
\end{aligned}
$$

In principle, we could endow $V_{A}^{r}$ with the standard graph norm in order to make $V_{A}^{r}$ a Hilbert space. However, we will choose an equivalent Hilbert structure later on. In the same way, for $\sigma>0$, we define the power $B^{\sigma}$ of $B$. For its domain, we use the notation

$$
\begin{aligned}
& V_{B}^{\sigma}:=D\left(B^{\sigma}\right), \text { with the norm }\|\cdot\|_{B, \sigma} \text { associated with the inner product } \\
& (v, w)_{B, \sigma}:=(v, w)+\left(B^{\sigma} v, B^{\sigma} w\right) \text { for } v, w \in V_{B}^{\sigma} .
\end{aligned}
$$

At this point, we can start listing our assumptions. First of all,

$$
r, \sigma_{0} \text { and } \tau \text { are fixed positive numbers, and } \sigma \in\left(0, \sigma_{0}\right) \text { is a parameter. }
$$

As for the linear operators, we postulate, besides (2.2), that

$$
\begin{aligned}
& \text { either } \lambda_{1}>0 \text { or } 0=\lambda_{1}<\lambda_{2} \text { and } e_{1} \text { is a constant; } \\
& \text { if } \lambda_{1}=0 \text {, then the constant functions belong to } V_{B}^{\sigma} \text {. }
\end{aligned}
$$

In [15], some remarks are given on the above assumptions. Moreover, it is shown that an equivalent Hilbert structure on $V_{A}^{r}$ is obtained by taking the norm defined by

$$
\|v\|_{A, r}^{2}:=\left\{\begin{array}{l}
\left\|A^{r} v\right\|^{2}=\sum_{j=1}^{\infty}\left|\lambda_{j}^{r}\left(v, e_{j}\right)\right|^{2} \quad \text { if } \lambda_{1}>0 \\
\left|\left(v, e_{1}\right)\right|^{2}+\left\|A^{r} v\right\|^{2}=\left|\left(v, e_{1}\right)\right|^{2}+\sum_{j=2}^{\infty}\left|\lambda_{j}^{r}\left(v, e_{j}\right)\right|^{2} \quad \text { if } \lambda_{1}=0
\end{array}\right.
$$

and the corresponding inner product, which we term $(\cdot, \cdot)_{A, r}$. This equivalence is trivial if $\lambda_{1}>0$. In the opposite case $\lambda_{1}=0$, with the notation

$$
\text { mean } v:=\frac{1}{|\Omega|} \int_{\Omega} v \quad \text { for } v \in L^{1}(\Omega)
$$

for the mean value of the generic function $v$, the equivalence relies on the inequality

$$
\|v\| \leq C_{P}\left\|A^{r} v\right\| \text { for every } v \in V_{A}^{r} \text { with mean } v=0 \text { if } \lambda_{1}=0
$$


which is of Poincaré type, since the term $\left(v, e_{1}\right)$ appearing in (2.11) and involving the constant function $e_{1}$ (see (2.9)) is proportional to mean $v$. Next, the nonlinear potential $F$ appearing in (1.2) is split as follows:

$$
\begin{aligned}
F= & \widehat{\beta}+\widehat{\pi}, \text { where } \\
& \widehat{\beta}: \mathbb{R} \rightarrow[0,+\infty] \text { is convex, proper and l.s.c. with } \widehat{\beta}(0)=0 \\
& \widehat{\pi}: \mathbb{R} \rightarrow \mathbb{R} \text { is of class } C^{1} \text { with a Lipschitz continuous first derivative; } \\
& \liminf s^{-2} F(s)>0 .
\end{aligned}
$$

Notice that these assumptions are fulfilled by all of the important potentials (1.4)(1.6). We set, for convenience,

$$
\beta:=\partial \widehat{\beta}, \quad \pi:=\widehat{\pi}^{\prime}, \quad \text { and } \quad L_{\pi}:=\text { the Lipschitz constant of } \pi \text {. }
$$

Moreover, we term $D(\widehat{\beta})$ and $D(\beta)$ the effective domains of $\widehat{\beta}$ and $\beta$, respectively, and notice that $\beta$ is a maximal monotone graph in $\mathbb{R} \times \mathbb{R}$. The same symbol $\beta$ is used for the maximal monotone operators induced in $L^{2}(\Omega)$ and $L^{2}(Q)$. Finally, we introduce

$$
P: H \rightarrow H, \quad \text { the orthogonal projection operator onto the kernel of } B \text {. (2.16) }
$$

As for the data of our problem, we allow the forcing term appearing in (1.2) to depend on $\sigma$ and assume that:

$$
\begin{aligned}
& f_{\sigma} \in L^{2}(0, T ; H) \\
& \varphi_{0} \in V_{B}^{\sigma_{0}} \text { and } \widehat{\beta}\left(\varphi_{0}\right) \in L^{1}(\Omega) ; \\
& \text { if } \lambda_{1}=0 \text { then } m_{0}:=\text { mean } \varphi_{0} \text { belongs to the interior of } D(\beta) .
\end{aligned}
$$

At this point, we make the notion of solution precise. In the following, we use the notations

$$
Q_{t}:=\Omega \times(0, T) \text { for } t \in(0, T] \text { and } Q:=Q_{T} .
$$

A solution to our system is a pair $\left(\varphi_{\sigma}, \mu_{\sigma}\right)$ fulfilling the regularity requirements

$$
\begin{aligned}
& \varphi_{\sigma} \in H^{1}(0, T ; H) \cap L^{\infty}\left(0, T ; V_{B}^{\sigma}\right), \\
& \mu_{\sigma} \in L^{2}\left(0, T ; V_{A}^{2 r}\right), \\
& \widehat{\beta}\left(\varphi_{\sigma}\right) \in L^{1}(Q),
\end{aligned}
$$


and satisfying the following weak formulation of Eqs. (1.1)-(1.3):

$$
\begin{aligned}
& \left(\partial_{t} \varphi_{\sigma}(t), v\right)+\left(A^{r} \mu_{\sigma}(t), A^{r} v\right)=0 \text { for every } v \in V_{A}^{r} \text { and for a.a. } t \in(0, T), \\
& \tau\left(\partial_{t} \varphi_{\sigma}(t), \varphi_{\sigma}(t)-v\right)+\left(B^{\sigma} \varphi_{\sigma}(t), B^{\sigma}\left(\varphi_{\sigma}(t)-v\right)\right) \\
& +\int_{\Omega} \widehat{\beta}\left(\varphi_{\sigma}(t)\right)+\left(\pi\left(\varphi_{\sigma}(t)\right)-f_{\sigma}(t), \varphi_{\sigma}(t)-v\right) \\
& \leq\left(\mu_{\sigma}(t), \varphi_{\sigma}(t)-v\right)+\int_{\Omega} \widehat{\beta}(v) \\
& \text { for every } v \in V_{B}^{\sigma} \text { and for a.a. } t \in(0, T) \text {, } \\
& \varphi_{\sigma}(0)=\varphi_{0} \text {. }
\end{aligned}
$$

We notice that (2.23) implies that $\widehat{\beta}\left(\varphi_{\sigma}(t)\right) \in L^{1}(\Omega)$ for a.a. $t \in(0, T)$, so that $(2.25)$ has a precise meaning. In the same inequality, one obviously has to read $\int_{\Omega} \widehat{\beta}(v)=$ $+\infty$ if $v \in V_{B}^{\sigma}$ and $\widehat{\beta}(v) \notin L^{1}(\Omega)$.

Remark 2.1. The regularity (2.22) of the second component of the solution is expected even though (2.24) just suggests $\mu_{\sigma} \in L^{2}\left(0, T ; V_{A}^{r}\right)$. Indeed, for a.a. $t \in(0, T)$ the variational equation has the form

$$
\left(A^{r} u, A^{r} v\right)=(g, v) \text { for every } v \in V_{A}^{r},
$$

with $g \in H$. From this, one easily derives that $u \in V_{A}^{2 r}$ and $\left\|A^{2 r} u\right\| \leq\|g\|$ (one can formally test by $A^{2 r} u$, but a regularization procedure makes the argument rigorous). Since $\partial_{t} \varphi_{\sigma} \in L^{2}(0, T ; H)$, we thus have the regularity (2.22) as well as

$$
\partial_{t} \varphi_{\sigma}+A^{2 r} \mu=0 \text { a.e. in }(0, T),
$$

i.e., the equation holds in its strong form.

Remark 2.2. In the sequel, the symbol 1 denotes the constant function on $\Omega$ that takes the value 1 at every point. With this notation, we remark that (2.9) implies that $A^{r}(\mathbf{1})$ vanishes if $\lambda_{1}=0$, so that (2.24) and (2.26) imply that

$$
\begin{aligned}
& \frac{\mathrm{d}}{\mathrm{d} t} \int_{\Omega} \varphi_{\sigma}(t)=0 \text { for a.a. } t \in(0, T), \text { whence } \\
& \text { mean } \varphi_{\sigma}(t)=m_{0} \text { for every } t \in[0, T]
\end{aligned}
$$

in this case. On the contrary, if $\lambda_{1}>0$, no conservation property is expected.

The well-posedness result (cf. [15, Thm. 2.6]) reads as follows:

Theorem 2.3. Let the assumptions (2.2), (2.8)-(2.10), and (2.14) on the structure of the system and (2.17)-(2.19) on the data be fulfilled. Then, there exists a pair $\left(\varphi_{\sigma}, \mu_{\sigma}\right)$ satisfying (2.21)-(2.23) and solving problem (2.24)-(2.26). Moreover, the component $\varphi_{\sigma}$ of the solution is unique. 
Remark 2.4. No uniqueness for the component $\mu_{\sigma}$ of the solution can be expected, in general. However, in particular situations, $\mu_{\sigma}$ is unique, too. This is the case if $\lambda_{1}>0$. Indeed, this assumption implies that $A^{2 r}$ is invertible so that (2.27) can be uniquely solved for $\mu_{\sigma}$. On the contrary, the case $\lambda_{1}=0$ is much more delicate. A sufficient condition that ensures uniqueness for $\mu_{\sigma}$ is the following (see [15, Rem. 4.1]): $\widehat{\beta}$ is an everywhere defined $C^{1}$ function and $\varphi_{\sigma}$ is bounded. We notice that the same argument used in the quoted remark also applies if $D(\beta)$ is an open interval and $\beta$ is a continuous single-valued function on it (like in the case (1.5) of the logarithmic potential) provided that all of the values of $\varphi_{\sigma}$ belong to a compact subset of $D(\beta)$.

Let us come to the results of this paper. The first deals with the behavior of the solutions to problem (2.24)-(2.26) as $\sigma$ tends to zero.

Theorem 2.5. Besides the assumptions of Theorem 2.3, assume that

$$
f_{\sigma} \rightarrow f \text { strongly in } L^{2}(0, T ; H) \text { as } \sigma \searrow 0 \text {. }
$$

Then, for every $\sigma>0$ there is a solution $\left(\varphi_{\sigma}, \mu_{\sigma}\right)$ to problem (2.24)-(2.26) such that

$$
\begin{aligned}
& \varphi_{\sigma} \rightarrow \varphi \text { weakly in } H^{1}(0, T ; H), \\
& \mu_{\sigma} \rightarrow \mu \text { weakly in } L^{2}\left(0, T ; V_{A}^{2 r}\right), \\
& B^{\sigma} \varphi_{\sigma} \rightarrow \zeta \text { weakly star in } L^{\infty}(0, T ; H),
\end{aligned}
$$

as $\sigma \searrow 0$, possibly along a subsequence, for some triplet $(\varphi, \mu, \zeta)$ satisfying

$$
\varphi \in H^{1}(0, T ; H), \quad \mu \in L^{2}\left(0, T ; V_{A}^{2 r}\right) \text { and } \zeta \in L^{\infty}(0, T ; H) .
$$

Moreover, under the additional assumption,

for all $v \in H$ such that $\widehat{\beta}(v) \in L^{1}(\Omega)$ there exists a sequence $\left\{v_{n}\right\} \subset V_{B}^{\sigma_{0}}$

$$
\text { such that } v_{n} \rightarrow v \text { in } H \text { and } \liminf _{n \rightarrow \infty} \int_{\Omega} \widehat{\beta}\left(v_{n}\right)=\int_{\Omega} \widehat{\beta}(v) \text {, }
$$

the following holds true: whenever $\left(\varphi_{\sigma}, \mu_{\sigma}\right)$ is a solution to problem (2.24)-(2.26) for $\sigma>0$ and (2.30)-(2.32) hold for some triplet $(\varphi, \mu, \zeta)$, then $\zeta=\varphi-P \varphi$ and the pair $(\varphi, \mu)$ is a solution to the system

$$
\begin{aligned}
& \left(\partial_{t} \varphi(t), v\right)+\left(A^{r} \mu(t), A^{r} v\right)=0 \text { for every } v \in V_{A}^{r} \text { and for a.a. } t \in(0, T), \\
& \tau\left(\partial_{t} \varphi(t), \varphi(t)-v\right)+(\varphi(t)-P \varphi(t), \varphi(t)-v) \\
& +\int_{\Omega} \widehat{\beta}(\varphi(t))+(\pi(\varphi(t))-f(t), \varphi(t)-v) \\
& \leq(\mu(t), \varphi(t)-v)+\int_{\Omega} \widehat{\beta}(v) \\
& \text { for every } v \in H \text { and for a.a. } t \in(0, T) \text {, } \\
& \varphi(0)=\varphi_{0} \text {. }
\end{aligned}
$$


Remark 2.6. The above statement looks a little involved. Besides the assumption (2.34) we are going to discuss in a while, we point out that no uniqueness for the solution $\left(\varphi_{\sigma}, \mu_{\sigma}\right)$ is required. On the contrary, if additional assumptions were made that guarantee uniqueness for $\left(\varphi_{\sigma}, \mu_{\sigma}\right)$ (see Remark 2.4) and (2.34) were assumed, then the statement would look much simpler, namely: as $\sigma$ tends to zero, the solution $\left(\varphi_{\sigma}, \mu_{\sigma}\right)$ converges (in the sense of (2.30)-(2.31), possibly along a subsequence) to a solution $(\varphi, \mu)$ to problem (2.35)-(2.37). If, in addition, uniqueness holds for the solution $(\varphi, \mu)$ to the limiting problem, then the whole family $\left\{\left(\varphi_{\sigma}, \mu_{\sigma}\right)\right\}$ converges to $(\varphi, \mu)$ as $\sigma$ tends to zero.

Remark 2.7. As observed in the forthcoming Remark 3.3, if (2.34) is not assumed, a weaker conclusion can anyway be obtained: the variational inequality (2.36) is fulfilled by all the test functions $v \in V_{B}^{\sigma_{0}}$. Indeed, it is stressed in the remark that assumption (2.34) is used in the proof of Theorem 2.5 just to extend to any $v \in H$ the validity of (2.36) proved for test functions $v \in V_{B}^{\sigma_{0}}$.

Remark 2.8. So, if (2.34) is assumed, then every limiting pair $(\varphi, \mu)$ satisfies (2.36) with arbitrary test functions $v \in H$. This has the following important consequence: there exists some $\xi$ satisfying

$$
\begin{aligned}
& \xi \in L^{2}(0, T ; H) \text { and } \xi \in \beta(\varphi) \text { a.e. in } Q, \\
& \tau \partial_{t} \varphi+\varphi-P \varphi+\xi+\pi(\varphi)=\mu+f \text { a.e. in } Q .
\end{aligned}
$$

Indeed, if we set

$$
\xi:=\mu-\tau \partial_{t} \varphi-\varphi+P \varphi-\pi(\varphi)+f
$$

then $\xi$ belongs to $L^{2}(0, T ; H)$, Eq. (2.39) is satisfied, and (2.36) becomes

$$
\begin{aligned}
& \int_{\Omega} \widehat{\beta}\left(\varphi(t) \leq(\xi, \varphi(t)-v)+\int_{\Omega} \widehat{\beta}(v)\right. \\
& \quad \text { for every } v \in H \text { and for a.a. } t \in(0, T) .
\end{aligned}
$$

But this exactly means that $\xi(t) \in \partial \widehat{\beta}(\varphi(t))=\beta(\varphi(t))$ for a.a. $t \in(0, T)$, i.e., the second condition in (2.38). If instead (2.34) is not assumed, then (2.36) is satisfied only for test functions $v \in V_{B}^{\sigma_{0}}$, as said in Remark 2.7. Nevertheless, the definition (2.40) still yields $\xi \in L^{2}(0, T ; H)$ and implies that (2.39) is satisfied. However, in this case, (2.41) is only true for $v \in V_{B}^{\sigma_{0}}$, and this means that for a.a. $t \in(0, T)$ the function $\xi(t)$ belongs to the subdifferential of the function $V_{B}^{\sigma_{0}} \ni v \mapsto \int_{\Omega} \widehat{\beta}(v)$. Notice that this subdifferential is a subset of the dual space $\left(V_{B}^{\sigma_{0}}\right)^{*}$ and might contain elements that do not belong to $H$ (in the sense of the Hilbert triplet $\left.\left(V_{B}^{\sigma_{0}}, H,\left(V_{B}^{\sigma_{0}}\right)^{*}\right)\right)$. Moreover, if a function $u \in H$ belongs to such a subdifferential, then it is not clear whether it also belongs to the subdifferential in $H$ (i.e., that of the function $H \ni v \mapsto \int_{\Omega} \widehat{\beta}(v)$ ), so that we cannot conclude that $\xi \in \beta(\varphi)$ a.e. in $Q$. About this matter, let us quote the paper [5] for related issues. 
Remark 2.9. A sufficient condition for (2.34) to hold true is the following (satisfied in all of the concrete cases, at least if $\sigma_{0}$ is small enough):

$$
H^{2}(\Omega) \subset V_{B}^{\sigma_{0}}
$$

In order to construct the sequence $\left\{v_{n}\right\}$ for a given $v \in H$, we solve the Neumann boundary value problem

$$
\int_{\Omega} v_{n} z+\frac{1}{n} \int_{\Omega} \nabla v_{n} \cdot \nabla z=\int_{\Omega} v z \text { for every } z \in H^{1}(\Omega) .
$$

Since $v \in H$, we have that $v_{n} \in H^{2}(\Omega)$ and thus $v_{n} \in V_{B}^{\sigma_{0}}$, by (2.42). Now, if we take $z=v_{n}$ in (2.43) and use the Cauchy-Schwarz inequality in the right-hand side, then we easily find that

$$
\left\|v_{n}\right\| \leq\|v\| \quad \text { and } \quad\left\|\frac{1}{n} \nabla v_{n}\right\|^{2} \leq \frac{1}{n}\|v\|^{2} \text { for all } n \in \mathbb{N} .
$$

Hence, there are a subsequence $\left\{v_{n_{k}}\right\}$ and some $w \in H$ such that $v_{n_{k}} \rightarrow w$ weakly in $H$. Moreover, since $\frac{1}{n} \nabla v_{n} \rightarrow(0,0,0)$ strongly in $H \times H \times H$ by $(2.44)$, we easily infer from (2.43) that $w=v$. A fortiori, by the uniqueness of the limit point, the entire sequence $\left\{v_{n}\right\}$ converges weakly in $H$ to $v$. But then, by the weak sequential lower semicontinuity of norms,

$$
\|v\| \leq \liminf _{n \rightarrow \infty}\left\|v_{n}\right\| \leq \limsup _{n \rightarrow \infty}\left\|v_{n}\right\| \leq\|v\|
$$

where the latter inequality follows from (2.44). We thus have $\|v\|=\lim _{n \rightarrow \infty}\left\|v_{n}\right\|$, and the uniform convexity of $H$ yields that $v_{n} \rightarrow v$ strongly in $H$.

Now, denoting by $\widehat{\beta}_{\varepsilon}$ and $\beta_{\varepsilon}$ the Moreau-Yosida $\varepsilon$-approximations of $\widehat{\beta}$ and $\beta$, respectively (see, e.g., [7, p. 28]), we account for the definition of the subdifferential $\beta_{\varepsilon}=\partial \widehat{\beta}_{\varepsilon}$ and the identity obtained by testing (2.43) by $\beta_{\varepsilon}\left(v_{n}\right) \in H^{1}(\Omega)$. We have that

$$
\int_{\Omega} \widehat{\beta}_{\varepsilon}\left(v_{n}\right)-\int_{\Omega} \widehat{\beta}_{\varepsilon}(v) \leq \int_{\Omega} \beta_{\varepsilon}\left(v_{n}\right)\left(v_{n}-v\right)=-\frac{1}{n} \int_{\Omega} \beta_{\varepsilon}^{\prime}\left(v_{n}\right)\left|\nabla v_{n}\right|^{2} \leq 0
$$

and we deduce that

$$
\int_{\Omega} \widehat{\beta}_{\varepsilon}\left(v_{n}\right) \leq \int_{\Omega} \widehat{\beta}_{\varepsilon}(v) \leq \int_{\Omega} \widehat{\beta}(v) \text { whence also } \int_{\Omega} \widehat{\beta}\left(v_{n}\right) \leq \int_{\Omega} \widehat{\beta}(v)
$$

by letting $\varepsilon$ tend to zero. This implies the inequality " $\leq$ " in (2.34). Since the opposite inequality clearly follows from the lower semicontinuity of the function $z \mapsto \int_{\Omega} \widehat{\beta}(z)$ in $H$, we finally deduce the validity of (2.34).

Notice that Theorem 2.3 ensures the existence of at least one solution to the limiting problem (2.35)-(2.37) with the regularity specified in (2.33). Our next result deals with partial uniqueness and continuous dependence of the solution. This will be proved in the last Sect. 4, which is devoted to the study of the limiting problem. 
Theorem 2.10. Let the general assumptions on the structure be fulfilled, and assume that $\varphi_{0}$ satisfies (2.18). Moreover, let $f_{i} \in L^{2}(0, T ; H), i=1,2$, be two choices of the forcing term $f$ appearing in (2.36), and let $\left(\varphi_{i}, \mu_{i}\right) \in H^{1}(0, T ; H) \times L^{2}\left(0, T ; V_{A}^{2 r}\right)$ be two corresponding solutions to problem (2.35)-(2.37) with $f=f_{i}$. Then, we have

$$
\left\|\varphi_{1}-\varphi_{2}\right\|_{L^{\infty}(0, T ; H)} \leq C_{c d}\left\|f_{1}-f_{2}\right\|_{L^{2}(0, T ; H)},
$$

with a constant $C_{c d}$ that depends only on $\tau$, the Lipschitz constant $L_{\pi}$, and $T$. In particular, if $f \in L^{2}(0, T ; H)$, the first component $\varphi$ of the solution $(\varphi, \mu)$ to problem (2.35)-(2.37) is uniquely determined.

In our final result, we require some regularity of the data and further assumptions on the structure that are satisfied in all of the concrete cases of interest, and we prove a regularity result. As a byproduct, we obtain a sufficient condition for the uniqueness of the second component $\mu$ of the solution. Sufficient conditions for uniqueness in a different direction are given in the forthcoming Remark 4.5.

Theorem 2.11. Let the general assumptions on the structure be fulfilled. In addition, assume that

$$
\begin{aligned}
& V_{B}^{n} \subset H^{1}(\Omega) \text { for some positive integer } n, \\
& V_{A}^{2 r} \subset H^{\eta}(\Omega), \quad f \in L^{2}\left(0, T ; H^{\eta}(\Omega)\right) \text { and } \varphi_{0} \in H^{\eta}(\Omega) \text { for some } \eta \in(0,1],
\end{aligned}
$$

and let $(\varphi, \mu)$ with

$$
\varphi \in H^{1}(0, T ; H) \text { and } \mu \in L^{2}\left(0, T ; V_{A}^{2 r}\right)
$$

be a solution to problem (2.35)-(2.37). Then, $\varphi$ enjoys the further regularity

$$
\varphi \in L^{2}\left(0, T ; H^{\eta}(\Omega)\right)
$$

and there exists some $\xi$ satisfying (2.38)-(2.39). In particular, even the second component $\mu$ of the solution is unique if $\beta$ is single-valued.

Throughout the paper, we widely use the Cauchy-Schwarz and Young inequalities, the latter in the form

$$
a b \leq \delta a^{2}+\frac{1}{4 \delta} b^{2} \text { for every } a, b \in \mathbb{R} \text { and } \delta>0 .
$$

Moreover, in performing a priori estimates, we use the same small letter $c$ for (possibly) different constants that depend only on the structure of our system but $\sigma$, and on the assumptions on the data. In particular, the values of $c$ do not depend on the regularization parameter $\lambda$ we introduce in the next section. On the contrary, some precise constants are denoted by different symbols (see, e.g., (2.13), where a capital letter with an index is used). 


\section{Asymptotic analysis}

This section is devoted to the proof of Theorem 2.5. The construction of the solutions $\left(\varphi_{\sigma}, \mu_{\sigma}\right)$ mentioned in the statement relies on a priori estimates on the solutions to a regularized problem, as done in [15] to solve problem (2.24)-(2.26) with a fixed $\sigma$. Hence, we briefly recall that regularization procedure. For $\lambda>0$ (small enough if needed), let $\beta_{\lambda}$ be the Yosida approximation of $\beta$ at the level $\lambda$ (see, e.g., [7, p. 28]). The corresponding Moreau regularization $\widehat{\beta_{\lambda}}$ of $\widehat{\beta}$ is thus given by

$$
\widehat{\beta}_{\lambda}(s)=\int_{0}^{s} \beta_{\lambda}\left(s^{\prime}\right) \mathrm{d} s^{\prime} \quad \text { for } s \in \mathbb{R}
$$

since $\beta_{\lambda}(0)=0$ due to (2.14). Then, the regularized problem is to find a pair $\left(\varphi_{\sigma}^{\lambda}, \mu_{\sigma}^{\lambda}\right)$ satisfying the regularity requirements

$$
\varphi_{\sigma}^{\lambda} \in H^{1}(0, T ; H) \cap L^{\infty}\left(0, T ; V_{B}^{\sigma}\right) \cap L^{2}\left(0, T ; V_{B}^{2 \sigma}\right) \text { and } \mu_{\sigma}^{\lambda} \in L^{2}\left(0, T ; V_{A}^{2 r}\right) \text {, }
$$

and solving the following system:

$\left(\partial_{t} \varphi_{\sigma}^{\lambda}(t), v\right)+\left(A^{r} \mu_{\sigma}^{\lambda}(t), A^{r} v\right)=0$ for every $v \in V_{A}^{r}$ and for a.a. $t \in(0, T)$,

$\tau\left(\partial_{t} \varphi_{\sigma}^{\lambda}(t), v\right)+\left(B^{\sigma} \varphi_{\sigma}^{\lambda}(t), B^{\sigma} v\right)+\left(\beta_{\lambda}\left(\varphi_{\sigma}^{\lambda}(t)\right)+\pi\left(\varphi_{\sigma}^{\lambda}(t)\right)-f_{\sigma}(t), v\right)$

$=\left(\mu_{\sigma}^{\lambda}(t), v\right)$ for every $v \in V_{B}^{\sigma}$ and for a.a. $t \in(0, T)$,

$\varphi_{\sigma}^{\lambda}(0)=\varphi_{0}$.

We notice that the variational inequality (2.25) is replaced by the equality (3.3) in the approximating problem (since $\beta_{\lambda}$ is an everywhere defined Lipschitz continuous function). The existence part of Theorem 2.3 is proved by solving the above regularized problem (cf. [15, Thm. 5.1]) and showing that its solution $\left(\varphi_{\sigma}^{\lambda}, \mu_{\sigma}^{\lambda}\right)$ converges as $\lambda \searrow 0$ (in a suitable topology, possibly just along a subsequence) to a pair $\left(\varphi_{\sigma}, \mu_{\sigma}\right)$ which turns out to solve problem (2.24)-(2.26). This solution, where now $\sigma$ is a varying parameter that we intend to approach zero, will be the good candidate for Theorem 2.5.

Before starting to estimate, it is worth observing that Remark 2.1 applies to both equations (3.2) and (3.3). This is obvious for the former. As far as the latter is concerned, one has to replace $A$ and $r$ by $B$ and $\sigma$, respectively, and notice that $\beta_{\lambda}$ is Lipschitz continuous, so that $\mu_{\sigma}^{\lambda}+f_{\sigma}-\beta_{\lambda}\left(\varphi_{\sigma}^{\lambda}\right)-\pi\left(\varphi_{\sigma}^{\lambda}\right) \in L^{2}(0, T ; H)$. This justifies the last regularity condition for $\varphi_{\sigma}^{\lambda}$ in (3.1) (in contrast to (2.21)) and implies the strong form of both equations, i.e.,

$$
\begin{aligned}
& \partial_{t} \varphi_{\sigma}^{\lambda}+A^{2 r} \mu_{\sigma}^{\lambda}=0 \quad \text { a.e. in }(0, T), \\
& \tau \partial_{t} \varphi_{\sigma}^{\lambda}+B^{2 \sigma} \varphi_{\sigma}^{\lambda}+\beta_{\lambda}\left(\varphi_{\sigma}^{\lambda}\right)+\pi\left(\varphi_{\sigma}^{\lambda}\right)=\mu_{\sigma}^{\lambda}+f_{\sigma} \quad \text { a.e. in }(0, T) .
\end{aligned}
$$

We also recall the convention on the symbol $c$ for possibly different constants made at the end of Sect. 2. Moreover, since (2.29) implies that $f_{\sigma}$ is bounded in $L^{2}(0, T ; H)$, we allow $c$ to also depend on a bound for the corresponding norm. 
First a priori estimate We test (3.2) written at the time $s$ by $\mu_{\sigma}(s)$. At the same time, we insert $+\varphi_{\sigma}^{\lambda}(s)$ to both sides of (3.6) written at the time $s$ and multiply it by $\partial_{t} \varphi_{\sigma}^{\lambda}(s)$, then integrating over $\Omega$. We sum up both equalities, noting that a cancellation occurs, and integrate over $(0, t)$ with respect to $s$. We obtain

$$
\begin{gathered}
\int_{0}^{t}\left\|A^{r} \mu_{\sigma}^{\lambda}(s)\right\|^{2} \mathrm{~d} s+\tau \int_{Q_{t}}\left|\partial_{t} \varphi_{\sigma}^{\lambda}\right|^{2}+\frac{1}{2}\left(\left\|\varphi_{\sigma}^{\lambda}(t)\right\|^{2}+\left\|B^{\sigma} \varphi_{\sigma}^{\lambda}(t)\right\|^{2}\right)+\int_{\Omega} \widehat{\beta_{\lambda}}\left(\varphi_{\sigma}^{\lambda}(t)\right) \\
=\frac{1}{2}\left(\left\|\varphi_{0}\right\|^{2}+\left\|B^{\sigma} \varphi_{0}\right\|^{2}\right)+\int_{\Omega} \widehat{\beta}_{\lambda}\left(\varphi_{0}\right)+\int_{Q_{t}}\left(f_{\sigma}+\varphi_{\sigma}^{\lambda}-\pi\left(\varphi_{\sigma}^{\lambda}\right)\right) \partial_{t} \varphi_{\sigma}^{\lambda} .
\end{gathered}
$$

Even the last integral on the left-hand side is nonnegative. We estimate the terms on the right-hand side by accounting for the assumptions (2.18) and (2.29) on $\varphi_{0}$ and $f_{\sigma}$, respectively, and owing to the Lipschitz continuity of $\pi$. Recalling also (2.7), we have that

$$
\begin{aligned}
\left\|\varphi_{0}\right\|^{2}+\left\|B^{\sigma} \varphi_{0}\right\|^{2}=\left\|\varphi_{0}\right\|_{B, \sigma}^{2}=\sum_{j=1}^{\infty}\left(1+\left(\lambda_{j}^{\prime}\right)^{2 \sigma}\right)\left|\left(\varphi_{0}, e_{j}^{\prime}\right)\right|^{2} \\
\leq \sum_{j=1}^{\infty}\left(2+\left(\lambda_{j}^{\prime}\right)^{2 \sigma_{0}}\right)\left|\left(\varphi_{0}, e_{j}^{\prime}\right)\right|^{2} \leq 2\left\|\varphi_{0}\right\|_{B, \sigma_{0}}^{2}, \\
\quad \int_{\Omega} \widehat{\beta}_{\lambda}\left(\varphi_{0}\right) \leq \int_{\Omega} \widehat{\beta}\left(\varphi_{0}\right), \\
\quad \int_{Q_{t}}\left(f_{\sigma}+\varphi_{\sigma}^{\lambda}-\pi\left(\varphi_{\sigma}^{\lambda}\right)\right) \partial_{t} \varphi_{\sigma}^{\lambda} \leq \frac{\tau}{2} \int_{Q_{t}}\left|\partial_{t} \varphi_{\sigma}^{\lambda}\right|^{2} \\
\quad+c \int_{0}^{t}\left(\left\|f_{\sigma}(s)\right\|^{2}+\left\|\varphi_{\sigma}^{\lambda}(s)\right\|^{2}+1\right) \mathrm{d} s \\
\leq \frac{\tau}{2} \int_{Q_{t}}\left|\partial_{t} \varphi_{\sigma}^{\lambda}\right|^{2}+c \int_{0}^{t}\left\|\varphi_{\sigma}^{\lambda}(s)\right\|^{2} \mathrm{~d} s+c .
\end{aligned}
$$

Therefore, by rearranging and applying the Gronwall lemma, we conclude that

$$
\left\|A^{r} \mu_{\sigma}^{\lambda}\right\|_{L^{2}(0, T ; H)}+\left\|\varphi_{\sigma}^{\lambda}\right\|_{H^{1}(0, T ; H)}+\left\|\varphi_{\sigma}^{\lambda}\right\|_{L^{\infty}\left(0, T ; V_{B}^{\sigma}\right)}+\left\|\widehat{\beta}_{\lambda}\left(\varphi_{\sigma}^{\lambda}\right)\right\|_{L^{\infty}\left(0, T ; L^{1}(\Omega)\right)} \leq c .
$$

From this and (3.5), we deduce that

$$
\left\|A^{2 r} \mu_{\sigma}^{\lambda}\right\|_{L^{2}(0, T ; H)} \leq c .
$$

Second a priori estimate Our aim is to improve the estimate concerning $\mu_{\sigma}^{\lambda}$. Indeed, for the following we need that

$$
\left\|\mu_{\sigma}^{\lambda}\right\|_{L^{2}\left(0, T ; V_{A}^{r}\right)} \leq c .
$$

We notice at once that this and (3.8) would imply that

$$
\left\|\mu_{\sigma}^{\lambda}\right\|_{L^{2}\left(0, T ; V_{A}^{2 r}\right)} \leq c .
$$


The desired estimate trivially follows from (3.7) if $\lambda_{1}>0$. So, we now deal with the other case $\lambda_{1}=0$ and apply a well-known trick based on the assumption (2.19) and the subsequent inequality

$$
\beta_{\lambda}(s)\left(s-m_{0}\right) \geq \delta_{0}\left|\beta_{\lambda}(s)\right|-C_{0},
$$

which holds for some $C_{0}>0$ and every $s \in \mathbb{R}$ and $\lambda \in(0,1)$, where $\delta_{0}$ is such that the interval $\left[m_{0}-\delta_{0}, m_{0}+\delta_{0}\right]$ is included in the interior of $D(\beta)$ (cf. [28, Appendix, Prop. A.1]; see also [22, p. 908] for a detailed proof). Inequality (3.11) implies that

$$
\left(\beta_{\lambda}\left(\varphi_{\sigma}^{\lambda}(t)\right), \varphi_{\sigma}^{\lambda}(t)-m_{0} \mathbf{1}\right) \geq \delta_{0}\left\|\beta_{\lambda}\left(\varphi_{\sigma}^{\lambda}(t)\right)\right\|_{L^{1}(\Omega)}-c \text { for a.a. } t \in(0, T),
$$

and this can be used when testing equation (3.3) by $\varphi_{\sigma}^{\lambda}-m_{0} \mathbf{1}$. To this concern, we recall that $\mathbf{1} \in V_{B}^{\sigma}$ by (2.10) and notice that the conservation property (2.28) also holds for $\varphi_{\sigma}^{\lambda}$, i.e., mean $\varphi_{\sigma}^{\lambda}(t)=m_{0}$ for every $t \in[0, T]$. So, for a.a. $t \in(0, T)$, we test (3.3) by $\varphi_{\sigma}^{\lambda}(t)-m_{0} \mathbf{1}$ and make some minor adjustments. However, we omit writing the time $t$ for a while. We also write $k$ instead of $k \mathbf{1}$ if $k$ is a real number. We have a.e. in $(0, T)$ that

$$
\begin{aligned}
\left\|B^{\sigma} \varphi_{\sigma}^{\lambda}\right\|^{2}+\left(\beta_{\lambda}\left(\varphi_{\sigma}^{\lambda}\right), \varphi_{\sigma}^{\lambda}-m_{0}\right) \\
\quad=\left(\mu_{\sigma}^{\lambda}, \varphi_{\sigma}^{\lambda}-m_{0}\right)+\left(f_{\sigma}-\tau \partial_{t} \varphi_{\sigma}^{\lambda}-\pi\left(\varphi_{\sigma}^{\lambda}\right), \varphi_{\sigma}^{\lambda}-m_{0}\right)+\left(B^{\sigma} \varphi_{\sigma}^{\lambda}, B^{\sigma} m_{0}\right) .
\end{aligned}
$$

The left-hand side of this equality can be estimated from below by virtue of (3.12). The first term on the right-hand side can be dealt with by accounting for the Poincaré-type inequality (2.13) as follows:

$$
\begin{aligned}
& \left(\mu_{\sigma}^{\lambda}, \varphi_{\sigma}^{\lambda}-m_{0}\right)=\left(\mu_{\sigma}^{\lambda}-\text { mean } \mu_{\sigma}^{\lambda}, \varphi_{\sigma}^{\lambda}-m_{0}\right) \leq \| \mu_{\sigma}^{\lambda}-\text { mean } \mu_{\sigma}^{\lambda}\|\| \varphi_{\sigma}^{\lambda}-m_{0} \| \\
& \leq c \| A^{r}\left(\mu_{\sigma}^{\lambda}-\text { mean } \mu_{\sigma}^{\lambda}\right)\|\| \varphi_{\sigma}^{\lambda}-m_{0}\|=c\| A^{r} \mu_{\sigma}^{\lambda}\|\| \varphi_{\sigma}^{\lambda}-m_{0} \|,
\end{aligned}
$$

the last equality being due to $A^{r} \mathbf{1}=0$. Therefore, by recalling (3.7), we have that the whole right-hand side of (3.13) is bounded in $L^{2}(0, T)$ and conclude that

$$
\left\|\beta_{\lambda}\left(\varphi_{\sigma}^{\lambda}\right)\right\|_{L^{2}\left(0, T ; L^{1}(\Omega)\right)} \leq c \text {, whence immediately } \| \text { mean } \beta_{\lambda}\left(\varphi_{\sigma}^{\lambda}\right) \|_{L^{2}(0, T)} \leq c .
$$

At this point, we can test the second equation (3.3) by $\mathbf{1}$ and deduce a bound for mean $\mu_{\sigma}^{\lambda}$ in $L^{2}(0, T)$. This and (3.7) imply (3.9). As already noticed, (3.10) is proved as well.

First conclusion As already remarked, in the proof of [15, Thm. 5.1] with a fixed $\sigma$ it is shown that $\left(\varphi_{\sigma}^{\lambda}, \mu_{\sigma}^{\lambda}\right)$ converges as $\lambda$ tends to zero (in a proper topology, possibly along a subsequence) to some pair $\left(\varphi_{\sigma}, \mu_{\sigma}\right)$, and it is proved that such a pair is a solution to problem (2.24)-(2.26). We prove that the family $\left\{\left(\varphi_{\sigma}, \mu_{\sigma}\right)\right\}_{\sigma>0}$ constructed in this way satisfies all the requirement of the statement. The starting point is the conservation of the bounds just proved in the limit as $\lambda \searrow 0$. We have that

$$
\left\|\varphi_{\sigma}\right\|_{H^{1}(0, T ; H)}+\left\|\mu_{\sigma}\right\|_{L^{2}\left(0, T ; V_{A}^{2 r}\right)}+\left\|B^{\sigma} \varphi_{\sigma}\right\|_{L^{\infty}(0, T ; H)} \leq c,
$$


and we conclude that (2.30)-(2.32) hold true for some triplet $(\varphi, \mu, \zeta)$ satisfying (2.33). This ends the proof of the first part of the statement.

Let us come to the second part. So, we assume that $\left\{\left(\varphi_{\sigma}, \mu_{\sigma}\right)\right\}_{\sigma>0}$ is a family of solutions to problem (2.24)-(2.26) and that (2.30)-(2.32) hold true for some triplet $(\varphi, \mu, \zeta)$ satisfying (2.33) as $\sigma \searrow 0$, possibly for a subsequence (however, we always write $\sigma$ instead of the elements of some subsequence $\left\{\sigma_{k}\right\}$, for brevity). We have to prove that $\zeta=\varphi-P \varphi$ and that $(\varphi, \mu)$ solves problem (2.35)-(2.37), by also assuming (2.34).

First characterization We are going to show that $\zeta=\varphi-P \varphi$ by proving that

$$
B^{\sigma} \varphi_{\sigma} \rightarrow \varphi-P \varphi \text { weakly in } L^{2}(Q) .
$$

To this end, we use the eigenvalues $\lambda_{j}^{\prime}$ and the eigenfunctions $e_{j}^{\prime}$ of $B$ and notice that $e_{j}^{\prime}$ is orthogonal to $\operatorname{ker} B$ if $\lambda_{j}^{\prime}>0$ while $\lambda_{j}^{\prime}=0$ if $e_{j}^{\prime} \in \operatorname{ker} B$. We set, for convenience,

$$
A_{j}^{\sigma}(\psi):=\int_{0}^{T}\left(B^{\sigma} \varphi_{\sigma}(t), \psi(t) e_{j}^{\prime}\right) \mathrm{d} t=\left(\lambda_{j}^{\prime}\right)^{\sigma} \int_{0}^{T}\left(\varphi_{\sigma}(t), \psi(t) e_{j}^{\prime}\right) \mathrm{d} t
$$

for $\psi \in L^{2}(0, T)$ and $j=1,2, \ldots$, and we notice that (3.14) follows if we prove that

$$
\lim _{\sigma \searrow 0} A_{j}^{\sigma}(\psi)=A_{j}^{0}(\psi):=\int_{0}^{T}\left(\varphi(t)-P \varphi(t), \psi(t) e_{j}^{\prime}\right) \mathrm{d} t
$$

for every $\psi$ and $j$ as before, since the linear combinations of the products $\psi e_{j}^{\prime}$ of such real functions and eigenfunctions of $B$ form a dense subspace of $L^{2}(Q)$. So, we fix $\psi$ and $j$. As for $j$, we distinguish two cases. Assume first that $\lambda_{j}^{\prime}>0$. Then, $\left(\lambda_{j}^{\prime}\right)^{\sigma}$ tends to 1 as $\sigma$ tends to zero. Moreover, (2.30) holds. We thus deduce that

$$
\lim _{\sigma \searrow 0} A_{j}^{\sigma}(\psi)=\int_{0}^{T}\left(\varphi(t), \psi(t) e_{j}^{\prime}\right) \mathrm{d} t=A_{j}^{0}(\psi),
$$

the last equality being due to the orthogonality between $P \varphi(t)$ and $e_{j}^{\prime}$. Assume now that $\lambda_{j}=0$. Then, we trivially have that $A_{j}^{\sigma}(\psi)=0$ for every $\sigma>0$. On the other hand, we also have that $A_{j}^{0}(\psi)=0$ since $e_{j}^{\prime} \in \operatorname{ker} B$ and $\varphi(t)-P \varphi(t)$ is orthogonal to $\operatorname{ker} B$ for a.a. $t \in(0, T)$. Therefore, $(3.15)$ is proved in any case.

Remark 3.1. The same argument shows that, for every $v \in L^{2}\left(0, T ; V_{B}^{\sigma_{0}}\right)$, the weak convergence $B^{\sigma} v \rightarrow v-P v$ in $L^{2}(0, T ; H)$ holds true as $\sigma$ tends to zero. In fact, the convergence is strong:

$$
B^{\sigma} v \rightarrow v-P v \quad \text { strongly in } L^{2}(0, T ; H) \text { for every } v \in L^{2}\left(0, T ; V_{B}^{\sigma_{0}}\right) .
$$

Indeed, for a.a. $t \in(0, T), B^{\sigma} v(t) \rightarrow v(t)-P v(t)$ strongly in $H$ by [13, Lem. 7.5]. Moreover, the Lebesgue dominated convergence theorem can be applied since

$$
\left\|B^{\sigma} v(t)\right\|^{2}=\sum_{j=1}^{\infty}\left(\lambda_{j}^{\prime}\right)^{2 \sigma}\left|\left(v(t), e_{j}^{\prime}\right)\right|^{2} \leq \sum_{j=1}^{\infty}\left(1+\left(\lambda_{j}^{\prime}\right)^{2 \sigma_{0}}\right)\left|\left(v(t), e_{j}^{\prime}\right)\right|^{2}=\|v(t)\|_{B, \sigma_{0}}^{2}
$$

for a.a. $t \in(0, T)$ and every $\sigma \in\left(0, \sigma_{0}\right]$, and $\|v(\cdot)\|_{B, \sigma_{0}}^{2}$ belongs to $L^{1}(0, T)$. 
To conclude the proof, we have to show that $(\varphi, \mu)$ solves problem (2.35)-(2.37) under the further assumption (2.34). The first equation obviously follows from (2.24) due to (2.30)-(2.31), and the initial condition (2.37) is satisfied as well since (2.30) implies weak convergence in $C^{0}([0, T] ; H)$. So, it remains to verify the variational inequality (2.36). To this concern, it is convenient to give different formulations of both (2.25) and (2.36). This procedure is based on the lemma stated below, which follows from the classical theory of variational inequalities of elliptic type in the framework of Convex Analysis.

Lemma 3.2. Let $V$ be a Hilbert space, $V^{*}$ its dual space, $\langle\cdot, \cdot\rangle$ the duality pairing between $V^{*}$ and $V$, and $a: V \times V \rightarrow \mathbb{R}$ a continuous bilinear form. Moreover, assume that

$\widehat{\gamma}_{1}: V \rightarrow(-\infty,+\infty]$ is convex, proper and lower semicontinuous,

$\widehat{\gamma}_{2}: V \rightarrow \mathbb{R}$ is convex and Gâteaux differentiable,

and $\gamma_{2}: V \rightarrow V^{*}$ is its Gâteaux derivative.

Then, for every $u \in V$ and $g \in V^{*}$, the variational inequalities

$$
\begin{aligned}
& a(u, u-v)+\widehat{\gamma}_{1}(u)+\left\langle\gamma_{2}(u), u-v\right\rangle \leq\langle g, u-v\rangle+\widehat{\gamma}_{1}(v) \text { for every } v \in V, \\
& a(u, u-v)+\widehat{\gamma}_{1}(u)+\widehat{\gamma}_{2}(u) \leq\langle g, u-v\rangle+\widehat{\gamma}_{1}(v)+\widehat{\gamma}_{2}(v) \text { for every } v \in V,
\end{aligned}
$$

are equivalent to each other.

As announced, we use this lemma to replace both (2.25) and (2.36) by different variational inequalities.

First alternative formulation We first observe that (2.25) for every $v \in V_{B}^{\sigma}$ as required implies the same inequality for every $v \in V_{B}^{\sigma_{0}}$ since $V_{B}^{\sigma_{0}} \subset V_{B}^{\sigma}$. Now, by recalling that $L_{\pi}$ is the Lipschitz constant of $\pi$, we replace the latter variational inequality by an equivalent one by applying the lemma above, with the choices

$$
\begin{gathered}
V=V_{B}^{\sigma_{0}}, \quad a(u, v)=\int_{\Omega}\left(B^{\sigma} u, B^{\sigma} v\right)-L_{\pi}(u, v) \quad \text { for } u, v \in V, \\
\widehat{\gamma}_{1}(v)=\int_{\Omega} \widehat{\beta}(v) \text { and } \widehat{\gamma}_{2}(v)=\int_{\Omega}\left(\widehat{\pi}(v)+\frac{L_{\pi}}{2} v^{2}\right) \text { for } v \in V, \\
\quad \text { and, for a.a. } t \in(0, T), \quad u=\varphi_{\sigma}(t) \text { and } g=\mu_{\sigma}(t)+f_{\sigma}(t)-\tau \mathrm{d} t \varphi_{\sigma}(t) .
\end{gathered}
$$

Notice that $\widehat{\gamma_{2}}$ actually is convex (since $\pi^{\prime}+L_{\pi} \geq 0$ a.e. in $\mathbb{R}$ ) and Gâteaux differentiable and that its derivative $\gamma_{2}$ is given by $\left\langle\gamma_{2}(u), v\right\rangle=\left(\pi(u)+L_{\pi} u, v\right)$. Hence, we deduce that the variational inequality (2.25) required just for every $v \in V_{B}^{\sigma_{0}}$ is 
equivalent to

$$
\begin{aligned}
& \tau\left(\partial_{t} \varphi_{\sigma}(t), \varphi_{\sigma}(t)-v\right)+\left(B^{\sigma} \varphi_{\sigma}(t), B^{\sigma}\left(\varphi_{\sigma}(t)-v\right)\right)-L_{\pi}\left(\varphi_{\sigma}(t), \varphi_{\sigma}(t)-v\right) \\
& \quad+\int_{\Omega} \widehat{\alpha}\left(\varphi_{\sigma}(t)\right) \leq\left(\mu_{\sigma}(t)+f_{\sigma}(t), \varphi_{\sigma}(t)-v\right)+\int_{\Omega} \widehat{\alpha}(v) \\
& \quad \text { for every } v \in V_{B}^{\sigma_{0}} \text { and for a.a. } t \in(0, T)
\end{aligned}
$$

where, for brevity, we have set

$$
\widehat{\alpha}(s):=\widehat{\beta}(s)+\widehat{\pi}(s)+\frac{L_{\pi}}{2} s^{2} \text { for } s \in \mathbb{R} .
$$

We fix what we have established:

the variational inequality (2.25) implies (3.21).

Second alternative formulation Similarly, we would like to show that (2.36) is equivalent to

$$
\begin{aligned}
& \tau\left(\partial_{t} \varphi(t), \varphi(t)-v\right)+(\varphi(t)-P \varphi(t), \varphi(t)-v)-L_{\pi}(\varphi(t), \varphi(t)-v) \\
& \quad+\int_{\Omega} \widehat{\alpha}(\varphi(t)) \leq(\mu(t)+f(t), \varphi(t)-v)+\int_{\Omega} \widehat{\alpha}(v) \\
& \quad \text { for every } v \in V_{B}^{\sigma_{0}} \text { and for a.a. } t \in(0, T) .
\end{aligned}
$$

Unfortunately, this does not seem to be true, in general, and we prove the following:

$$
\text { the variational inequality (2.36) with } v \text { varying in } V_{B}^{\sigma_{0}} \text { is equivalent to (3.24). (3.25) }
$$

To this aim, it suffices to apply the lemma with the same $\widehat{\gamma_{i}}$ as before and obvious $u$ and $g$, but with $V=V_{B}^{\sigma_{0}}$ and $a$ defined by $a(u, v):=(u-P u, v)-L_{\pi}(u, v)$ for $u, v \in V_{B}^{\sigma_{0}}$.

Conclusion of the proof In view of (3.23) and (3.25), our aim is first to verify (3.24) by starting from (3.21) (implied by (2.25)), while (2.36), as it is, will be proved at the end by accounting for (2.34). However, the left-hand side of (3.21) contains the quadratic term associated with the map $v \mapsto-L_{\pi} \int_{\Omega}|v|^{2}$. This term is unpleasant since the related map is concave. To get rid of it, we adapt the procedure introduced in [13] to the present case. We set, for convenience,

$$
\kappa:=\frac{L_{\pi}}{\tau}, \quad \rho_{\sigma}(t):=e^{-\kappa t} \varphi_{\sigma}(t) \text { and } \rho(t):=e^{-\kappa t} \varphi(t) \text { for a.a. } t \in(0, T),
$$

and we notice that $w \mapsto \int_{Q} e^{-2 \kappa t} w^{2}$ is the square of an equivalent norm in $L^{2}(Q)$. At this point, we pick an arbitrary $v \in L^{2}\left(0, T ; V_{B}^{\sigma_{0}}\right)$, write (3.21) by taking $v(t)$ as 
test function, multiply by $e^{-2 \kappa t}$, and integrate over $(0, T)$. We obtain

$$
\begin{gathered}
\int_{0}^{T} \tau\left(e^{-\kappa t}\left(\partial_{t} \varphi_{\sigma}(t)-\kappa \varphi_{\sigma}(t)\right), e^{-\kappa t}\left(\varphi_{\sigma}-v\right)(t)\right) \mathrm{d} t+\int_{0}^{T} e^{-2 \kappa t}\left\|B^{\sigma} \varphi_{\sigma}(t)\right\|^{2} \mathrm{~d} t \\
\quad-\int_{0}^{T} e^{-2 \kappa t}\left(B^{\sigma} \varphi_{\sigma}(t), B^{\sigma} v(t)\right) \mathrm{d} t+\int_{Q} e^{-2 \kappa t} \widehat{\alpha}\left(\varphi_{\sigma}\right) \\
\leq \int_{0}^{T} e^{-2 \kappa t}\left(\mu_{\sigma}(t)+f_{\sigma}(t),\left(\varphi_{\sigma}-v\right)(t)\right) \mathrm{d} t+\int_{Q} e^{-2 \kappa t} \widehat{\alpha}(v)
\end{gathered}
$$

Well, we want to take the limit as $\sigma$ tends to zero in this inequality. As for the first term on the left-hand side, we have that

$$
\begin{aligned}
\int_{0}^{T} \tau\left(e^{-\kappa t}\left(\partial_{t} \varphi_{\sigma}(t)-\kappa \varphi_{\sigma}(t)\right), e^{-\kappa t}\left(\varphi_{\sigma}-v\right)(t)\right) \mathrm{d} t= & \int_{0}^{T} \tau\left(\partial_{t} \rho_{\sigma}(t), \rho_{\sigma}(t)-e^{-\kappa t} v(t)\right) \mathrm{d} t \\
= & \frac{\tau}{2}\left\|\rho_{\sigma}(T)\right\|^{2}-\frac{\tau}{2}\left\|\varphi_{0}\right\|^{2} \\
& -\int_{0}^{T} \tau\left(\partial_{t} \rho_{\sigma}(t), e^{-\kappa t} v(t)\right) \mathrm{d} t .
\end{aligned}
$$

By observing that $\rho_{\sigma}$ converges to $\rho$ weakly in $H^{1}(0, T ; H)$, thus weakly in $C^{0}([0, T] ; H)$, so that $\rho_{\sigma}(T)$ converges to $\rho(T)$ weakly in $H$, we therefore have that

$$
\begin{gathered}
\liminf _{\sigma \searrow 0} \int_{0}^{T} \tau\left(e^{-\kappa t}\left(\partial_{t} \varphi_{\sigma}(t)-\kappa \varphi_{\sigma}(t)\right), e^{-\kappa t}\left(\varphi_{\sigma}-v\right)(t)\right) \mathrm{d} t \\
\geq \frac{\tau}{2}\|\rho(T)\|^{2}-\frac{\tau}{2}\left\|\varphi_{0}\right\|^{2}-\int_{0}^{T} \tau\left(\partial_{t} \rho(t), e^{-\kappa t} v(t)\right) \mathrm{d} t \\
\quad=\int_{0}^{T} \tau\left(e^{-\kappa t}\left(\partial_{t} \varphi(t)-\kappa \varphi(t)\right), e^{-\kappa t}(\varphi-v)(t)\right) \mathrm{d} t
\end{gathered}
$$

Next, by (3.14) and the lower semicontinuity of the norms, we have that

$$
\liminf _{\sigma \searrow 0} \int_{0}^{T} e^{-2 \kappa t}\left\|B^{\sigma} \varphi_{\sigma}(t)\right\|^{2} \mathrm{~d} t \geq \int_{0}^{T} e^{-2 \kappa t}\|(\varphi-P \varphi)(t)\|^{2} \mathrm{~d} t .
$$

By also recalling (3.16), we can write

$$
\lim _{\sigma \searrow 0} \int_{0}^{T} e^{-2 \kappa t}\left(B^{\sigma} \varphi_{\sigma}(t), B^{\sigma} v(t)\right) \mathrm{d} t=\int_{0}^{T} e^{-2 \kappa t}((\varphi-P \varphi)(t),(v-P v)(t)) \mathrm{d} t .
$$

By taking the difference, we deduce that

$$
\begin{aligned}
& \underset{\sigma \searrow 0}{\liminf }\left(\int_{0}^{T} e^{-2 \kappa t}\left\|B^{\sigma} \varphi_{\sigma}(t)\right\|^{2} \mathrm{~d} t-\int_{0}^{T} e^{-2 \kappa t}\left(B^{\sigma} \varphi_{\sigma}(t), B^{\sigma} v(t)\right) \mathrm{d} t\right) \\
& \geq \int_{0}^{T} e^{-2 \kappa t}\|(\varphi-P \varphi)(t)\|^{2} \mathrm{~d} t-\int_{0}^{T} e^{-2 \kappa t}((\varphi-P \varphi)(t),(v-P v)(t)) \\
& \quad=\int_{0}^{T} e^{-2 \kappa t}((\varphi-P \varphi)(t),(\varphi-P \varphi)(t)-(v-P v)(t)) \mathrm{d} t \\
& =\int_{0}^{T} e^{-2 \kappa t}((\varphi-P \varphi)(t),(\varphi-v)(t)) \mathrm{d} t,
\end{aligned}
$$


the last equality being due to the orthogonality between $(\varphi-P \varphi)(t) \in(\operatorname{ker} B)^{\perp}$ and $(P \varphi-P v)(t) \in \operatorname{ker} B$. Moreover, by observing that the functional $w \mapsto \int_{Q} e^{-2 \kappa t} \widehat{\alpha}(w)$ is lower semicontinuous on $L^{2}(Q)$, and recalling that $\varphi_{\sigma}$ converges to $\varphi$ weakly in $L^{2}(Q)$, we deduce that

$$
\liminf _{\sigma \searrow 0} \int_{Q} e^{-2 \kappa t \widehat{\alpha}}\left(\varphi_{\sigma}\right) \geq \int_{Q} e^{-2 \kappa t} \widehat{\alpha}(\varphi) .
$$

This ends the treatment of the terms on the left-hand side of (3.27). Concerning the right-hand side, we have to overcome the difficulty due to the coupling between $\mu_{\sigma}$ and $\varphi_{\sigma}$. To this end, we introduce the notation

$$
(1 * w)(t):=\int_{0}^{t} w(s) \mathrm{d} s \text { for every } w \in L^{2}(0, T ; H) \text { and } t \in[0, T]
$$

and deduce from (2.27) that

$$
\varphi_{\sigma}+A^{2 r}\left(1 * \mu_{\sigma}\right)=\varphi_{0} .
$$

Hence, we have that

$$
\begin{aligned}
& \int_{0}^{T} e^{-2 \kappa t}\left(\mu_{\sigma}(t),\left(\varphi_{\sigma}-v\right)(t)\right) \mathrm{d} t=\int_{0}^{T} e^{-2 \kappa t}\left(\mu_{\sigma}(t), \varphi_{0}\right) \mathrm{d} t \\
& -\int_{0}^{T} e^{-2 \kappa t}\left(A^{r} \mu_{\sigma}(t), A^{r}\left(1 * \mu_{\sigma}\right)(t)\right) \mathrm{d} t-\int_{0}^{T} e^{-2 \kappa t}\left(\mu_{\sigma}(t), v(t)\right) \mathrm{d} t .
\end{aligned}
$$

Now, from (2.31) we deduce that $1 * \mu_{\sigma}$ converges to $1 * \mu$ weakly in $H^{1}\left(0, T ; V_{A}^{2 r}\right)$. Since the embedding $H^{1}\left(0, T ; V_{A}^{2 r}\right) \subset L^{2}\left(0, T ; V_{A}^{r}\right)$ is compact, we infer that $1 *$ $\mu_{\sigma}$ converges to $1 * \mu$ strongly in $L^{2}\left(0, T ; V_{A}^{r}\right)$. In view of (2.35) and (2.37), we deduce that

$$
\begin{aligned}
\lim _{\sigma \searrow 0} & \int_{0}^{T} e^{-2 \kappa t}\left(\mu_{\sigma}(t),\left(\varphi_{\sigma}-v\right)(t)\right) \mathrm{d} t=\int_{0}^{T} e^{-2 \kappa t}\left(\mu(t), \varphi_{0}\right) \mathrm{d} t \\
& -\int_{0}^{T} e^{-2 \kappa t}\left(A^{r} \mu(t), A^{r}(1 * \mu)(t)\right) \mathrm{d} t-\int_{0}^{T} e^{-2 \kappa t}(\mu(t), v(t)) \mathrm{d} t . \\
& =\int_{0}^{T} e^{-2 \kappa t}(\mu(t),(\varphi-v)(t)) \mathrm{d} t .
\end{aligned}
$$

Finally, by recalling (2.29), we see that the term involving $f_{\sigma}$ and the last one of (3.27) do not give any trouble. Therefore, we conclude that

$$
\begin{aligned}
& \int_{0}^{T} \tau\left(e^{-\kappa t}\left(\partial_{t} \varphi(t)-\kappa \varphi(t)\right), e^{-\kappa t}(\varphi-v)(t)\right) \mathrm{d} t \\
& \quad+\int_{0}^{T} e^{-2 \kappa t}((\varphi-P \varphi)(t),(\varphi-v)(t)) \mathrm{d} t+\int_{Q} e^{-2 \kappa t} \widehat{\alpha}\left(\varphi_{\sigma}\right) \\
& \quad \leq \int_{0}^{T} e^{-2 \kappa t}(\mu(t)+f(t),(\varphi-v)(t)) \mathrm{d} t+\int_{Q} e^{-2 \kappa t} \widehat{\alpha}(v),
\end{aligned}
$$


and this holds for every $v \in L^{2}\left(0, T ; V_{B}^{\sigma_{0}}\right)$. On the other hand, (3.28) is equivalent to

$$
\begin{aligned}
& \tau\left(e^{-\kappa t}\left(\partial_{t} \varphi(t)-\kappa \varphi(t)\right), e^{-\kappa t}(\varphi(t)-v)(t)\right) \\
& \quad+e^{-2 \kappa t}((\varphi-P \varphi)(t), \varphi(t)-v)+\int_{Q} e^{-2 \kappa t} \widehat{\alpha}\left(\varphi_{\sigma}\right) \\
& \leq e^{-2 \kappa t}(\mu(t)+f(t), \varphi(t)-v)+\int_{\Omega} e^{-2 \kappa t} \widehat{\alpha}(v)
\end{aligned}
$$

for a.a. $t \in(0, T)$ and every $v \in V_{B}^{\sigma_{0}}$. By multiplying by $e^{2 \kappa t}$ and recalling that $\kappa=L_{\pi} / \tau$, we obtain (3.24) as claimed. Recalling (3.25), we have proved that the variational inequality (2.36) is satisfied for every test function $v \in V_{B}^{\sigma_{0}}$. At this point, we account for (2.34), not yet used up to now, and show that (2.36) actually holds for every $v \in H$. To this end, for a given $v \in H$ with $\widehat{\beta}(v) \in L^{1}(\Omega)$ without loss of generality, it suffices to take a sequence $\left\{v_{n}\right\}$ given by (2.34), test (3.24) by $v_{n}$ and let $n$ tend to infinity. One obtains (3.24) for $v$ without any trouble. This completes the proof.

Remark 3.3. Going back to the above proof, one justifies what has been announced in Remark 2.7: if (2.34) is not assumed, one anyway arrives at the variational inequality (2.36) required for every $v \in V_{B}^{\sigma_{0}}$ instead of for every $v \in H$. Indeed, (2.34) has been only used at the end, in order to extend to any $v \in H$ the validity of (2.36) already proved for test functions $v \in V_{B}^{\sigma_{0}}$.

\section{The limiting problem}

In this section, we prove Theorems 2.10 and 2.11. As far as the former is concerned, some preliminaries are needed. We refer to [15, Sect. 3] for more details. We set

$$
V_{A}^{-r}:=\left(V_{A}^{r}\right)^{*} \text { and }\|\cdot\|_{A,-r}:=\text { the dual norm of }\|\cdot\|_{A, r}
$$

and we use the symbol $\langle\cdot, \cdot\rangle_{A, r}$ for the duality pairing between $V_{A}^{-r}$ and $V_{A}^{r}$. It is understood that $H$ is identified with a subspace of $V_{A}^{-r}$ in the usual way, i.e., in order that $\langle v, w\rangle_{A, r}=(v, w)$ for every $v \in H$ and $w \in V_{A}^{r}$. Moreover, we introduce the subspaces $V_{0}^{ \pm r}$ of $V_{A}^{ \pm r}$ by setting

$$
\begin{aligned}
V_{0}^{r} & :=V_{A}^{r} \text { and } V_{0}^{-r}:=V_{A}^{-r} \text { if } \lambda_{1}>0, \\
V_{0}^{r} & :=\left\{v \in V_{A}^{r}: \text { mean } v=0\right\} \text { and } V_{0}^{-r} \\
& :=\left\{\psi \in V_{A}^{-r}:\langle\psi, 1\rangle_{A, r}=0\right\} \quad \text { if } \lambda_{1}=0 .
\end{aligned}
$$

Next, we define $A_{0}^{2 r}: V_{0}^{r} \rightarrow V_{A}^{-r}$ by the formula

$$
\left\langle A_{0}^{2 r} v, w\right\rangle_{A, r}=\left(A^{r} v, A^{r} w\right)_{A, r} \quad \text { for every } v \in V_{0}^{r} \text { and } w \in V_{A}^{r} \text {. }
$$


It turns out that the range of $A_{0}^{2 r}$ is $V_{0}^{-r}$ and that $A_{0}^{2 r}$ is an isomorphism between $V_{0}^{r}$ and $V_{0}^{-r}$. Thus, we can set $A_{0}^{-2 r}:=\left(A_{0}^{2 r}\right)^{-1}$ and obtain an isomorphism between $V_{0}^{-r}$ and $V_{0}^{r}$. It also turns out that

$$
\left(A^{r} A_{0}^{-2 r} \psi, A^{r} v\right)=\langle\psi, v\rangle_{A, r} \quad \text { for every } \psi \in V_{0}^{-r} \text { and } v \in V_{A}^{r} .
$$

Finally, the following formula holds true:

$\left\langle\partial_{t} \psi, A_{0}^{-2 r} \psi\right\rangle_{A, r}=\frac{1}{2} \frac{\mathrm{d}}{\mathrm{d} t}\|\psi\|_{A,-r}^{2} \quad$ a.e. in $(0, T)$, for every $\psi \in H^{1}\left(0, T ; V_{0}^{-r}\right)$.

In particular,

$$
\int_{0}^{t}\left\langle\partial_{t} \psi(s), A_{0}^{-2 r} \psi(s)\right\rangle_{A, r} \mathrm{~d} s \geq 0 \text { for every } \psi \in H^{1}\left(0, T ; V_{0}^{-r}\right) \text { with } \psi(0)=0 \text {. }
$$

Proof of Theorem 2.10. We just prove the continuous dependence part, since uniqueness for the first component follows as a consequence. We set, for convenience, $f:=f_{1}-f_{2}, \varphi:=\varphi_{1}-\varphi_{2}$, and $\mu:=\mu_{1}-\mu_{2}$. Now, we write equation (2.35) at the time $s$ for these solutions and take the difference. Then, we test the resulting identity by $v=A_{0}^{-2 r} \varphi(s)$, where we observe that $\varphi(s) \in V_{0}^{-r}$, since $\varphi \in C^{0}([0, T] ; H)$ by (2.33) and mean $\varphi(s)=0$ if $\lambda_{1}=0$ by the conservation property (2.28), so that $v$ is a well-defined element of $V_{A}^{r}$. Moreover, we have that $A_{0}^{-2 r} \varphi \in L^{\infty}\left(0, T ; V_{A}^{r}\right)$. Integrating over $(0, t)$ with respect to $s$, where $t \in(0, T)$ is arbitrary, we obtain the identity

$$
\int_{0}^{t}\left\langle\partial_{t} \varphi(s), A_{0}^{-2 r} \varphi(s)\right\rangle_{A, r} \mathrm{~d} s+\int_{0}^{t}\left(A^{r} \mu(s), A^{r} A_{0}^{-2 r} \varphi(s)\right) \mathrm{d} s=0 .
$$

Now, the first term on the left-hand side is nonnegative by (4.2). Hence, by also noting that $\mu \in L^{2}\left(0, T ; V_{A}^{r}\right)$ and applying (4.1), we deduce that

$$
\int_{0}^{t}(\varphi(s), \mu(s)) \mathrm{d} s \leq 0 .
$$

At the same time, we write (2.36) for $f_{i}$ and $\left(\varphi_{i}, \mu_{i}\right), i=1,2$, test them by $\varphi_{2}$ and $\varphi_{1}$, respectively, add the resulting inequalities to each other, and integrate over $(0, t)$ as before. Then, the terms involving $\widehat{\beta}$ cancel out. By denoting by $I$ the identity map of $H$ and rearranging, we have that

$$
\begin{aligned}
& \frac{\tau}{2}\|\varphi(t)\|^{2}+\int_{0}^{t}((I-P) \varphi(s), \varphi(s)) \mathrm{d} s \\
& \quad \leq \int_{0}^{t}(f(s)+\mu(s), \varphi(s)) \mathrm{d} s-\int_{0}^{t}\left(\pi\left(\varphi_{1}(s)\right)-\pi\left(\varphi_{2}(s)\right), \varphi(s)\right) \mathrm{d} s .
\end{aligned}
$$

We observe that $I-P$ is the projection operator on the orthogonal subspace $(\operatorname{ker} B)^{\perp}$. It follows that $((I-P) v, v)=((I-P) v,(I-P) v) \geq 0$ for every $v \in H$, so that the 
second term on the left-hand side of (4.4) is nonnegative. By adding (4.3) and (4.4) to each other, and accounting for this observation, an obvious cancellation, the Lipschitz continuity of $\pi$ and the Schwarz and Young inequalities, we deduce that

$$
\frac{\tau}{2}\|\varphi(t)\|^{2} \leq \frac{1}{4} \int_{0}^{t}\|f(s)\|^{2} \mathrm{~d} s+\left(1+L_{\pi}\right) \int_{0}^{t}\|\varphi(s)\|^{2} \mathrm{~d} s .
$$

By applying the Gronwall lemma, we conclude that the desired estimate (2.45) holds true with a constant $C_{c d}$ as in the statement.

Finally, we prove Theorem 2.11. The proof we give is based on the study of the auxiliary problem of finding $\phi \in H^{1}(0, T ; H)$ satisfying

$$
\begin{aligned}
& \tau\left(\partial_{t} \phi(t), \phi(t)-v\right)+(\phi(t)-P \phi(t), \phi(t)-v) \\
& \quad+\int_{\Omega} \widehat{\beta}(\phi(t))+(\pi(\phi(t))-\pi(0), \phi(t)-v) \\
& \quad \leq(g(t), \phi(t)-v)+\int_{\Omega} \widehat{\beta}(v) \text { for every } v \in H \text { and for a.a. } t \in(0, T), \\
& \phi(0)=\phi_{0}
\end{aligned}
$$

for given

$$
g \in L^{2}(0, T ; H) \text { and } \phi_{0} \in H .
$$

We have subtracted the constant $\pi(0)$ from $\pi(\phi(t))$ in (4.5) in order to use the inequality $|\pi(s)-\pi(0)| \leq L_{\pi}|s|$ for $s \in \mathbb{R}$ without any additive constant. This is needed in the sequel, indeed. Since $\widehat{\beta}$ is convex, $P$ is linear and $\pi$ is Lipschitz continuous, this problem has a unique solution $\phi$ provided that the initial datum also satisfies

$$
\widehat{\beta}\left(\phi_{0}\right) \in L^{1}(\Omega) .
$$

In the forthcoming Lemma 4.2, we prove a regularity result by applying a particular case of [29, Sect. I, Thm. 2] which we present here in the form of a lemma.

Lemma 4.1. Let $\mathcal{A}_{0}, \mathcal{A}_{1}, \mathcal{B}_{0}$ and $\mathcal{B}_{1}$ be four Banach spaces with the continuous embeddings $\mathcal{A}_{0} \subset \mathcal{A}_{1}$ and $\mathcal{B}_{0} \subset \mathcal{B}_{1}$, and let $\mathcal{T}: \mathcal{A}_{1} \rightarrow \mathcal{B}_{1}$ be a nonlinear operator satisfying $\mathcal{T} v \in \mathcal{B}_{0}$ for every $v \in \mathcal{A}_{0}$. Assume that

$$
\begin{aligned}
& \|\mathcal{T} u-\mathcal{T} v\|_{\mathcal{B}_{1}} \leq C_{1}\|u-v\|_{\mathcal{A}_{1}} \text { for every } u, v \in \mathcal{A}_{1}, \\
& \|\mathcal{T} v\|_{\mathcal{B}_{0}} \leq C_{2}\|v\|_{\mathcal{A}_{0}} \text { for every } v \in \mathcal{A}_{0},
\end{aligned}
$$

for some positive constants $C_{1}$ and $C_{2}$. Then, for every $\vartheta \in(0,1)$ and $p \in[1,+\infty]$, we have that

$$
\begin{aligned}
& \mathcal{T} v \in\left(\mathcal{B}_{0}, \mathcal{B}_{1}\right)_{\vartheta, p} \text { and }\|\mathcal{T} v\|_{\left(\mathcal{B}_{0}, \mathcal{B}_{1}\right)_{\vartheta, p}} \leq C C_{1}^{\vartheta} C_{2}^{1-\vartheta}\|v\|_{\left(\mathcal{A}_{0}, \mathcal{A}_{1}\right)_{\vartheta, p}} \\
& \quad \text { for every } v \in\left(\mathcal{A}_{0}, \mathcal{A}_{1}\right)_{\vartheta, p},
\end{aligned}
$$

with a constant $C$ that does not depend on $\mathcal{T}$. 
In the above lemma, the symbol $\|\cdot\|_{X}$ stands for the norm in the generic Banach space $X$. The same convention is followed in the rest of the section, where $\|\cdot\|_{X}$ also denotes the norm in the power $X^{3}$ (however, we keep the short notation $\|\cdot\|$ without indices if $X=H)$. Moreover, $(X, Y)_{\vartheta, p}$ is the real interpolation space between the Banach spaces $X$ and $Y$ (for basic definitions and properties see, e.g., [27, Sect. 1.1]).

Lemma 4.2. Let the general assumption on the structure be fulfilled and assume that the data $g$ and $\phi_{0}$ satisfy

$$
g \in L^{2}\left(0, T ; H^{\eta}(\Omega)\right) \text { and } \phi_{0} \in H^{\eta}(\Omega)
$$

for some $\eta \in(0,1]$, as well as (4.8). Then, the solution $\phi$ to problem (4.5)-(4.6) enjoys the further regularity

$$
\phi \in L^{2}\left(0, T ; H^{\eta}(\Omega)\right),
$$

and there exists some $\xi$ satisfying

$$
\begin{aligned}
& \xi \in L^{2}(0, T ; H) \text { and } \xi \in \beta(\phi) \text { a.e. in } Q, \\
& \tau \partial_{t} \phi+\phi-P \phi+\xi+\pi(\phi)-\pi(0)=g \text { a.e. in } Q .
\end{aligned}
$$

Proof. By still denoting by $\widehat{\beta_{\lambda}}$ and $\beta_{\lambda}$ the Moreau-Yosida approximations of $\widehat{\beta}$ and $\beta$, respectively, we introduce the approximating problem of finding $\phi_{\lambda} \in H^{1}(0, T ; H)$ that satisfies

$$
\tau \partial_{t} \phi_{\lambda}+\phi_{\lambda}-P \phi_{\lambda}+\beta_{\lambda}\left(\phi_{\lambda}\right)+\pi\left(\phi_{\lambda}\right)-\pi(0)=g \text { a.e. in } Q
$$

and the initial condition (4.6). For any data satisfying (4.7) (while (4.8) is not needed here), also this problem has a unique solution $\phi_{\lambda}$. We perform some a priori estimates. As usual, the symbol $c$ stands for possibly different constants. In this proof, the values of $c$ can only depend on $\tau, \pi, \Omega, T$ and the eigenfunctions $e_{j}^{\prime}$ associated with the zero eigenvalues of $B$ (if any). In particular, they do not depend on $\lambda$, nor on the data of problem (4.5)-(4.6). Symbols like $C$ and $C_{i}$ denote particular values of $c$ we want to refer to. The first three estimates we perform are in the direction of the inequalities (4.9) and (4.10) which we want to satisfy with a suitable choice of the spaces and the operator. For this reason, they are obtained under different regularity assumptions on the data.

First a priori estimate Let $g_{i}$ and $\phi_{0, i}, i=1,2$, be two choices of the data satisfying (4.7) and let $\phi_{\lambda, i}$ be the corresponding solutions to the approximating problem. We set for brevity $\phi_{\lambda}:=\phi_{\lambda, 1}-\phi_{\lambda, 2}, g:=g_{1}-g_{2}$ and $\phi_{0}:=\phi_{0,1}-\phi_{0,2}$. We write (4.16) for both solutions, take the difference and multiply it by $\phi_{\lambda}$. Then, we integrate over $Q_{t}$. We obtain that

$$
\begin{aligned}
& \frac{\tau}{2} \int_{\Omega}\left|\phi_{\lambda}(t)\right|^{2}+\int_{Q_{t}}\left|\phi_{\lambda}\right|^{2}+\int_{Q_{t}}\left(\beta_{\lambda}\left(\phi_{\lambda, 1}\right)-\beta_{\lambda}\left(\phi_{\lambda, 2}\right)\right) \phi_{\lambda} \\
& \quad=\frac{\tau}{2} \int_{\Omega}\left|\phi_{0}\right|^{2}+\int_{Q_{t}} g \phi_{\lambda}+\int_{Q_{t}}\left(P \phi_{\lambda}\right) \phi_{\lambda}-\int_{Q_{t}}\left(\pi\left(\phi_{\lambda, 1}\right)-\pi\left(\phi_{\lambda, 2}\right)\right) \phi_{\lambda} .
\end{aligned}
$$


Since $\beta_{\lambda}$ is monotone, all of the terms on the left-hand side are nonnegative. By estimating the right-hand side on account of the Lipschitz continuity of $\pi$ and the Schwarz and Young inequalities, and then applying the Gronwall lemma, we easily conclude that

$$
\left\|\phi_{\lambda, 1}-\phi_{\lambda, 2}\right\|_{L^{\infty}(0, T ; H)} \leq C_{1, \infty}\left(\left\|g_{1}-g_{2}\right\|_{L^{2}(0, T ; H)}+\left\|\phi_{0,1}-\phi_{0,2}\right\|\right) .
$$

It trivially follows that

$$
\left\|\phi_{\lambda, 1}-\phi_{\lambda, 2}\right\|_{L^{2}(0, T ; H)} \leq C_{1}\left(\left\|g_{1}-g_{2}\right\|_{L^{2}(0, T ; H)}+\left\|\phi_{0,1}-\phi_{0,2}\right\|\right) .
$$

Second a priori estimate We assume (4.7) on the data. By multiplying (4.16) by $\phi_{\lambda}$ and integrating over $Q_{t}$, we obtain that

$$
\begin{aligned}
& \frac{\tau}{2} \int_{\Omega}\left|\phi_{\lambda}(t)\right|^{2}+\int_{Q_{t}}\left|\phi_{\lambda}\right|^{2}+\int_{Q_{t}} \beta_{\lambda}\left(\phi_{\lambda}\right) \phi_{\lambda} \\
& \quad=\frac{\tau}{2} \int_{\Omega}\left|\phi_{0}\right|^{2}+\int_{Q_{t}} g \phi_{\lambda}+\int_{Q_{t}}\left(P \phi_{\lambda}\right) \phi_{\lambda}+\int_{Q_{t}}\left(\pi\left(\phi_{\lambda}\right)-\pi(0)\right) \phi_{\lambda} .
\end{aligned}
$$

All of the terms on the left-hand side are nonnegative since $\beta_{\lambda}$ is monotone and $\beta_{\lambda}(0)=0$. If we estimate the right-hand side by using the Lipschitz continuity of $\pi$ and the Schwarz and Young inequality, we immediately deduce that

$$
\left\|\phi_{\lambda}\right\|_{L^{\infty}(0, T ; H)} \leq c\left(\|g\|_{L^{2}(0, T ; H)}+\left\|\phi_{0}\right\|\right) .
$$

Third a priori estimate We set $V:=H^{1}(\Omega)$ for brevity and assume that the data satisfy $g \in L^{2}(0, T ; V)$ and $\phi_{0} \in V$. Before going on, we make an observation. Assume first that ker $B=\{0\}$. Then, $P=0$ and (4.5) is an ordinary differential equation where the space variable is just a parameter. In the opposite case, the presence of the nonlocal operator $P$ could be unpleasant. However, we are reduced to the same situations as before by moving the term $P \phi_{\lambda}$ to the right-hand side and treating it as a datum. More precisely, in this case, $\operatorname{ker} B$ has a finite dimension $m>0$ and is spanned by the first $m$ eigenfunctions (those corresponding to the zero eigenvalues). Since every eigenfunction of $B$ belongs to the domain $V_{B}^{n}$ of $B^{n}$ for every $n \in \mathbb{N}$ and we are assuming (2.46), the eigenfunctions (we are interested in) belong to $V$, and we have the identities

$$
P v=\sum_{j=1}^{m}\left(v, e_{j}^{\prime}\right) e_{j}^{\prime} \quad \text { and } \quad \nabla P v=\sum_{j=1}^{m}\left(v, e_{j}^{\prime}\right) \nabla e_{j}^{\prime} \quad \text { for every } v \in H
$$

Namely, we have that $P v \in V$ even though $v$ only belongs to $H$. Therefore, in any case, the solution $\phi_{\lambda}$ enjoys some space regularity. Precisely, it belongs to $L^{2}(0, T ; V)$ as well as its time derivative and we have that

$$
\tau \partial_{t} \nabla \phi_{\lambda}+\nabla \phi_{\lambda}+\beta_{\lambda}^{\prime}\left(\phi_{\lambda}\right) \nabla \phi_{\lambda}+\pi^{\prime}\left(\phi_{\lambda}\right) \nabla \phi_{\lambda}=\nabla g+\nabla P \phi_{\lambda} \quad \text { a.e. in } Q \text {. }
$$


By multiplying this equation by $\nabla \phi_{\lambda}$ and integrating over $Q_{t}$, we obtain that

$$
\begin{aligned}
& \frac{\tau}{2} \int_{\Omega}\left|\nabla \phi_{\lambda}(t)\right|^{2}+\int_{Q_{t}}\left|\nabla \phi_{\lambda}\right|^{2}+\int_{Q_{t}} \beta_{\lambda}^{\prime}\left(\phi_{\lambda}\right)\left|\nabla \phi_{\lambda}\right|^{2} \\
& \quad=\frac{\tau}{2} \int_{\Omega}\left|\nabla \phi_{0}\right|^{2}+\int_{Q_{t}} \nabla g \cdot \nabla \phi_{\lambda}+\int_{Q_{t}}\left(\nabla P \phi_{\lambda}\right) \cdot \nabla \phi_{\lambda}-\int_{Q_{t}} \pi^{\prime}\left(\phi_{\lambda}\right)\left|\nabla \phi_{\lambda}\right|^{2} .
\end{aligned}
$$

All of the terms on the left-hand side are nonnegative. The volume integrals on the righthand side, except the one involving $P$, can be easily treated thanks to the boundedness of $\pi^{\prime}$ and the Schwarz and Young inequalities. If $P=0$, then we can apply the Gronwall lemma and obtain an estimate of $\nabla \phi_{\lambda}$. Recalling (4.19), we conclude that

$$
\left\|\phi_{\lambda}\right\|_{L^{\infty}(0, T ; V)} \leq C_{2, \infty}\left(\|g\|_{L^{2}(0, T ; V)}+\left\|\phi_{0}\right\|_{V}\right) .
$$

We claim that the same estimate holds true even though $\operatorname{ker} B$ is nontrivial. In this case, we recall the representation formula (4.20) and apply it to $\phi_{\lambda}$. By also accounting for standard inequalities, we obtain that

$$
\begin{aligned}
& \int_{Q_{t}}\left(\nabla P \phi_{\lambda}\right) \cdot \nabla \phi_{\lambda}=\int_{Q_{t}} \sum_{j=1}^{m}\left(\phi_{\lambda}, e_{j}^{\prime}\right) \nabla e_{j}^{\prime} \cdot \nabla \phi_{\lambda} \\
& =\sum_{j=1}^{m} \int_{0}^{t}\left(\left(\phi_{\lambda}(s), e_{j}^{\prime}\right) \int_{\Omega} \nabla e_{j}^{\prime} \cdot \nabla \phi_{\lambda}(s)\right) \mathrm{d} s \\
& \leq \sum_{j=1}^{m} \int_{0}^{t}\left\|\phi_{\lambda}(s)\right\|\left\|e_{j}^{\prime}\right\|\left\|\nabla e_{j}^{\prime}\right\|\left\|\nabla \phi_{\lambda}(s)\right\| \mathrm{d} s \leq c \int_{0}^{t}\left\|\phi_{\lambda}(s)\right\|\left\|\nabla \phi_{\lambda}(s)\right\| \mathrm{d} s \\
& \leq c\left\|\phi_{\lambda}\right\|_{L^{2}(0, T ; H)}^{2}+c \int_{Q_{t}}\left|\nabla \phi_{\lambda}\right|^{2} .
\end{aligned}
$$

So, it suffices to recall (4.19) and apply the Gronwall lemma to obtain (4.21) also in this case. Therefore, (4.21) is established and it trivially implies that

$$
\left\|\phi_{\lambda}\right\|_{L^{2}(0, T ; V)} \leq C_{2}\left(\|g\|_{L^{2}(0, T ; V)}+\left\|\phi_{0}\right\|_{V}\right) .
$$

Interpolation Now, let the data satisfy (4.12) with $\eta \in(0,1)$. We choose

$$
\begin{aligned}
& \mathcal{A}_{0}:=L^{2}(0, T ; V) \times V, \quad \mathcal{A}_{1}:=L^{2}(0, T ; H) \times H, \\
& \mathcal{B}_{0}:=L^{2}(0, T ; V) \text { and } \mathcal{B}_{1}:=L^{2}(0, T ; H)
\end{aligned}
$$

and apply Lemma 4.1 to the operator $\mathcal{T}: \mathcal{A}_{1} \rightarrow \mathcal{B}_{1}$ that associates to the pair $\left(g, \phi_{0}\right)$ the solution $\phi_{\lambda}$ to problem (4.5)-(4.6). Then, (4.18) and (4.22) yield (4.9) and (4.10), respectively. Moreover, by setting $\vartheta:=1-\eta$, we have that

$$
\begin{aligned}
\left(\mathcal{A}_{0}, \mathcal{A}_{1}\right)_{\vartheta, 2} & =\left(L^{2}(0, T ; V), L^{2}(0, T ; H)\right)_{\vartheta, 2} \times(V, H)_{\vartheta, 2} \\
& =L^{2}\left(0, T ; H^{\eta}(\Omega)\right) \times H^{\eta}(\Omega)
\end{aligned}
$$


so that $\left(g, \phi_{0}\right) \in\left(\mathcal{A}_{0}, \mathcal{A}_{1}\right)_{\vartheta, 2}$ by (4.12). It follows that

$$
\begin{aligned}
& \phi_{\lambda} \in\left(\mathcal{B}_{0}, \mathcal{B}_{1}\right)_{\vartheta, 2}=L^{2}\left(0, T ; H^{\eta}(\Omega)\right) \text { and } \\
& \left\|\phi_{\lambda}\right\|_{L^{2}\left(0, T ; H^{\eta}(\Omega)\right)} \leq C C_{1}^{\vartheta} C_{2}^{1-\vartheta}\left\|\left(g, \phi_{0}\right)\right\|_{L^{2}\left(0, T ; H^{\eta}(\Omega)\right) \times H^{\eta}(\Omega)}
\end{aligned}
$$

with a constant $C$ that does not depend on $\lambda$. Notice that (4.23) with $\eta=1$ (i.e., $\vartheta=0$ ) is ensured by (4.22).

Fourth a priori estimate We are close to the conclusion, and we thus assume that the data $g$ and $\phi_{0}$ are as in the statement. By multiplying (4.16) by $\partial_{t} \phi_{\lambda}$, integrating over $Q_{t}$, and rearranging, we have that

$$
\begin{aligned}
& \tau \int_{Q_{t}}\left|\partial_{t} \phi_{\lambda}\right|^{2}+\frac{1}{2} \int_{\Omega}\left|\phi_{\lambda}(t)\right|^{2}+\int_{\Omega} \widehat{\beta}_{\lambda}\left(\phi_{\lambda}(t)\right) \\
& \quad=\frac{1}{2} \int_{\Omega}\left|\phi_{0}\right|^{2}+\int_{\Omega} \widehat{\beta_{\lambda}}\left(\phi_{0}\right)+\int_{Q_{t}}\left(g+P \phi_{\lambda}-\pi\left(\phi_{\lambda}\right)+\pi(0)\right) \partial_{t} \phi_{\lambda} .
\end{aligned}
$$

Since $\widehat{\beta}_{\lambda}$ is nonnegative and $\widehat{\beta}_{\lambda}\left(\phi_{0}\right) \leq \widehat{\beta}\left(\phi_{0}\right)$ a.e. in $\Omega$, owing to the Schwarz and Young inequalities and the Lipschitz continuity of $\pi$, and accounting for (4.8) and (4.19), we infer that

$$
\left\|\partial_{t} \phi_{\lambda}\right\|_{L^{2}(0, T ; H)} \leq c\left(\|g\|_{L^{2}(0, T ; H)}+\left\|\phi_{0}\right\|+\left\|\widehat{\beta}\left(\phi_{0}\right)\right\|_{L^{1}(\Omega)}^{1 / 2}\right) .
$$

A comparison in (4.16) then yields that

$$
\left\|\beta_{\lambda}\left(\phi_{\lambda}\right)\right\|_{L^{2}(0, T ; H)} \leq c\left(\|g\|_{L^{2}(0, T ; H)}+\left\|\phi_{0}\right\|+\left\|\widehat{\beta}\left(\phi_{0}\right)\right\|_{L^{1}(\Omega)}^{1 / 2}\right) .
$$

Conclusion At this point, we let $\lambda$ tend to zero based on (4.23)-(4.25), the compact embedding $H^{\eta}(\Omega) \subset H$ for $\eta \in(0,1]$, and the well-known Aubin-Lions lemma (see, e.g., [26, Thm. 5.1, p. 58]). We deduce that there exists a pair $(\phi, \xi)$ such that

$$
\begin{aligned}
& \phi_{\lambda} \rightarrow \phi \quad \text { weakly star in } H^{1}(0, T ; H) \cap L^{2}\left(0, T ; H^{\eta}(\Omega)\right) \\
& \text { and strongly in } L^{2}(0, T ; H), \\
& \beta_{\lambda}\left(\phi_{\lambda}\right) \rightarrow \xi \text { weakly in } L^{2}(0, T ; H),
\end{aligned}
$$

possibly only for a subsequence $\lambda_{k} \searrow 0$. Then, $\phi(0)=\phi_{0}$, and (4.15) is verified. Moreover, by also applying, e.g., [4, Lemma 2.3, p. 38], we infer that $(\phi, \xi)$ satisfies the inclusion in (4.14). On the other hand, all this implies (4.5) since $\widehat{\beta}$ is convex, so that $\phi$ is the solution to problem (4.5)-(4.6). This completes the proof of the lemma.

Proof of Theorem 2.11. We apply Lemma 4.2 by choosing

$$
g=\mu+f-\pi(0) \text { and } \phi_{0}=\varphi_{0} .
$$

Notice that conditions (4.12) are satisfied due to (2.47)-(2.48). We thus obtain the existence of some $\xi$ satisfying (4.14) and (4.15). The latter reads

$$
\tau \partial_{t} \phi+\phi-P \phi+\xi+\pi(\phi)-\pi(0)=\mu+f-\pi(0) \text { a.e. in } Q \text {. }
$$


But $\varphi$ satisfies this equation (see Remark 2.8) since $(\varphi, \mu)$ is a solution to problem (2.35) $-(2.37)$ by assumption, and this implies (4.5) for $\varphi$ since $\widehat{\beta}$ is convex. On the other hand, we have that $\varphi(0)=\varphi_{0}=\phi_{0}$. Since the solution $\phi$ to problem (4.5)-(4.6) is unique, we conclude that $\phi=\varphi$. Therefore, (2.38)-(2.39) are proved. The last sentence of the statement trivially follows.

Remark 4.3. We observe that in Theorem 2.11 we start from a solution $(\varphi, \mu)$ to problem (2.35)-(2.37) without using sufficient conditions for the existence of such a solution. In particular, (2.34) is not accounted for. We also notice that the argument followed in the above proof provides the existence of a unique solution $\varphi$ to both equation (2.39) and the variational inequality (2.36) for a given $\mu$ without the use of (2.34).

Remark 4.4. It is possible to slightly modify the proof of Lemma 4.2 in the application of Lemma 4.1 and to obtain different regularity results in Theorem 2.11. One can play with the index $p$ in the interpolation argument, indeed. If we want to maximize the time regularity, we change the choice of the spaces $\mathcal{B}_{i}$ by taking

$$
\mathcal{B}_{0}:=L^{\infty}(0, T ; V) \text { and } \mathcal{B}_{1}:=L^{\infty}(0, T ; H)
$$

and start from (4.17) and (4.21) in place of (4.18) and (4.22). Then, we apply Lemma 4.1 still with $\vartheta=1-\eta$, but with $p=\infty$. Instead of (4.23), we obtain that

$$
\begin{aligned}
& \phi_{\lambda} \in\left(L^{\infty}(0, T ; V), L^{\infty}(0, T ; H)\right)_{\vartheta, \infty} \text { and } \\
& \quad\left\|\phi_{\lambda}\right\|_{\left(L^{\infty}(0, T ; V), L^{\infty}(0, T ; H)\right)_{\vartheta, \infty} \leq C C_{1}^{\vartheta} C_{2}^{1-\vartheta}\left\|\left(g, \phi_{0}\right)\right\|_{L^{2}\left(0, T ; H^{\eta}(\Omega)\right) \times H^{\eta}(\Omega)},}
\end{aligned}
$$

still with a constant $C$ that does not depend on $\lambda$. Then, everything can proceed as before. At the end of the proof of Theorem 2.11, we arrive at the regularity

$$
\varphi \in\left(L^{\infty}(0, T ; V), L^{\infty}(0, T ; H)\right)_{\vartheta, \infty}
$$

for the first component $\varphi$ of the solution $(\varphi, \mu)$ to problem (2.35)-(2.37). We avoid the troubles that may arise with the exponent $\infty$ and do not offer a different representation of the space appearing in (4.28). We just remark that the regularity (4.28) is neither better nor worse than (2.38), since it yields some better time regularity at the expense of a lower space regularity. One can prove that $\left(L^{\infty}(0, T ; V), L^{\infty}(0, T ; H)\right)_{\vartheta, \infty} \subset$ $L^{\infty}\left(0, T ; H^{\eta-\varepsilon}(\Omega)\right.$ ) for every $\varepsilon>0$ (in particular, the Aubin-Lions lemma can be applied also in the modified proof of Lemma 4.2) so that the Sobolev-type regularity for $\varphi$ we can obtain is

$$
\varphi \in L^{\infty}\left(0, T ; H^{\eta-\varepsilon}(\Omega)\right) \text { for every } \varepsilon>0 .
$$

Remark 4.5. Concerning uniqueness for the second component $\mu$ of the solution to problem (2.35)-(2.37), we can give sufficient conditions in a different direction. The situation is similar to the one encountered for problem (2.24)-(2.26) and mentioned in 
Remark 2.4. Let us give some detail. Assume that $\left(\varphi, \mu_{i}\right), i=1,2$, are solutions corresponding to some data $\varphi_{0}$ and $f$ (with the same first component, due to Theorem 2.10). By writing (2.35) for both solutions and taking the difference, we immediately obtain that $\left(A^{r}\left(\mu_{1}-\mu_{2}\right), v\right)=0$ for every $v \in V_{A}^{r}$ and a.e. in $(0, T)$, that is

$$
A^{r}\left(\mu_{1}-\mu_{2}\right)=0 \text {. }
$$

This implies that $\mu_{1}=\mu_{2}$ if $\lambda_{1}>0$. In the opposite case $\lambda_{1}=0$, we can arrive at the same conclusion under additional conditions, as we show at once by following the ideas of [15, Rem. 4.1]. However, in the present case, the condition we assume on the solutions is difficult to verify, unfortunately. Suppose that $D(\beta)$ is an open interval, the restriction of $\widehat{\beta}$ to $D(\beta)$ is a $C^{1}$ function, and all of the values attained by $\varphi$ belong to a compact subinterval $[a, b] \subset D(\beta)$. Now, choose $\delta_{0}$ such that the interval $\left[a-\delta_{0}, b+\delta_{0}\right]$ is contained in $D(\beta)$. Then, for an arbitrary $\delta \in\left(0, \delta_{0}\right)$ and for a.a. $t \in(0, T)$, we can choose $v=\varphi(t)-\delta$ (whence $\varphi(t)-v=\delta)$ and $v=\varphi(t)+\delta$ (whence $\varphi(t)-v=-\delta$ ) in the variational inequality (2.36) written for $\left(\varphi, \mu_{1}\right)$ and $\left(\varphi, \mu_{2}\right)$, respectively. Then, by adding the resulting inequalities, we deduce that

$$
2 \int_{\Omega} \widehat{\beta}(\varphi) \leq \delta\left(\mu_{1}-\mu_{2}, \mathbf{1}\right)+\int_{\Omega} \widehat{\beta}(\varphi-\delta)+\int_{\Omega} \widehat{\beta}(\varphi+\delta) \text { a.e. in }(0, T) .
$$

Division by $\delta$ then yields that

$$
\int_{\Omega} \frac{\widehat{\beta}(\varphi)-\widehat{\beta}(\varphi-\delta)}{\delta}+\int_{\Omega} \frac{\widehat{\beta}(\varphi)-\widehat{\beta}(\varphi+\delta)}{\delta} \leq\left(\mu_{1}-\mu_{2}, \mathbf{1}\right) .
$$

Taking the limit as $\delta \searrow 0$, we conclude from the Lebesgue dominated convergence theorem that

$$
0=\int_{\Omega} \beta(\varphi)-\int_{\Omega} \beta(\varphi) \leq\left(\mu_{1}-\mu_{2}, \mathbf{1}\right) .
$$

Interchanging the roles of $\mu_{1}$ and $\mu_{2}$, we then infer that mean $\mu_{1}=$ mean $\mu_{2}$ a.e. in $(0, T)$. By combining this with (4.29), we conclude that $\mu_{1}=\mu_{2}$.

\section{Acknowledgements}

This research was supported by the Italian Ministry of Education, University and Research (MIUR): Dipartimenti di Eccellenza Program (2018-2022)—Dept. of Mathematics "F. Casorati," University of Pavia. In addition, PC gratefully mentions some other support from the GNAMPA (Gruppo Nazionale per l'Analisi Matematica, la Probabilità e le loro Applicazioni) of INdAM (Istituto Nazionale di Alta Matematica), and PC and CG point out their affiliations as Research Associates to the IMATIC.N.R. Pavia, Italy.

Funding Open access funding provided by Università degli Studi di Pavia within the CRUI-CARE Agreement. 
Open Access. This article is licensed under a Creative Commons Attribution 4.0 International License, which permits use, sharing, adaptation, distribution and reproduction in any medium or format, as long as you give appropriate credit to the original author(s) and the source, provide a link to the Creative Commons licence, and indicate if changes were made. The images or other third party material in this article are included in the article's Creative Commons licence, unless indicated otherwise in a credit line to the material. If material is not included in the article's Creative Commons licence and your intended use is not permitted by statutory regulation or exceeds the permitted use, you will need to obtain permission directly from the copyright holder. To view a copy of this licence, visit http://creativecommons.org/licenses/ by/4.0/.

Publisher's Note Springer Nature remains neutral with regard to jurisdictional claims in published maps and institutional affiliations.

\section{REFERENCES}

[1] M. Ainsworth, Z. Mao, Analysis and approximation of a fractional Cahn-Hilliard equation, SIAM J. Numer. Anal. 55 (2017), 1689-1718.

[2] G. Akagi, G. Schimperna, A. Segatti, Fractional Cahn-Hilliard, Allen-Cahn and porous medium equations, J. Differential Equations 261 (2016), 2935-2985.

[3] G. Akagi, G. Schimperna, A. Segatti, Convergence of solutions for the fractional Cahn-Hilliard system, J. Funct. Anal. 276 (2019), 2663-2715.

[4] V. Barbu, "Nonlinear Differential Equations of Monotone Type in Banach Spaces", Springer, London, New York, 2010.

[5] V. Barbu, P. Colli, G. Gilardi, M. Grasselli, Existence, uniqueness, and longtime behavior for a nonlinear Volterra integrodifferential equation, Differential Integral Equations 13 (2000), 12331262.

[6] E. Bonetti, P. Colli, L. Scarpa, G. Tomassetti, Bounded solutions and their asymptotics for a doubly nonlinear Cahn-Hilliard system, Calc. Var. Partial Differential Equations, 59 (2020), Paper No. 88, 25 pp.

[7] H. Brezis, "Opérateurs maximaux monotones et semi-groupes de contractions dans les espaces de Hilbert”, North-Holland Math. Stud. 5, North-Holland, Amsterdam, 1973.

[8] L. Bu, L. Mei, Y. Hou, Stable second-order schemes for the space-fractional Cahn-Hilliard and Allen-Cahn equations, Comput. Math. Appl. 78 (2019), 3485-3500.

[9] C. Cavaterra, E. Rocca, H. Wu, Long-time dynamics and optimal control of a diffuse interface model for tumor growth, Appl. Math. Optim. 83 (2021), 739-787.

[10] R. Chill, E. Fašangová, J. Prüss, Convergence to steady state of solutions of the Cahn-Hilliard and Caginalp equations with dynamic boundary conditions, Math. Nachr. 279 (2006), 1448-1462.

[11] B. Climent-Ezquerra, F. Guillén-González, Convergence to equilibrium of global weak solutions for a Cahn-Hilliard-Navier-Stokes vesicle model, Z. Angew. Math. Phys. 70 (2019), Paper No. 125, 27 pp.

[12] P. Colli, T. Fukao, Nonlinear diffusion equations as asymptotic limits of Cahn-Hilliard systems, $J$. Differential Equations 260 (2016), 6930-6959.

[13] P. Colli, G. Gilardi, Well-posedness, regularity and asymptotic analyses for a fractional phase field system, Asympt. Anal. 114 (2019), 93-128.

[14] P. Colli, G. Gilardi, J. Sprekels, On the longtime behavior of a viscous Cahn-Hilliard system with convection and dynamic boundary conditions, J. Elliptic Parabol. Equ. 4 (2018), 327-347.

[15] P. Colli, G. Gilardi, J. Sprekels, Well-posedness and regularity for a generalized fractional CahnHilliard system, Atti Accad. Naz. Lincei Rend. Lincei Mat. Appl. 30 (2019), 437-478.

[16] P. Colli, G. Gilardi, J. Sprekels, Recent results on well-posedness and optimal control for a class of generalized fractional Cahn-Hilliard systems, Control Cybernet. 48 (2019), 153-197.

[17] P. Colli, G. Gilardi, J. Sprekels, Optimal distributed control of a generalized fractional Cahn-Hilliard system, Appl. Math. Optim. 82 (2020), 551-589.

[18] P. Colli, G. Gilardi, J. Sprekels, Asymptotic analysis of a tumor growth model with fractional operators, Asymptot. Anal. 120 (2020), 41-72. 
[19] P. Colli, G. Gilardi, J. Sprekels, Longtime behavior for a generalized Cahn-Hilliard system with fractional operators, Atti Accad. Peloritana Pericolanti Cl. Sci. Fis. Mat. Natur. 98 (2020), suppl. 2, A4, $18 \mathrm{pp}$.

[20] C.G. Gal, Well-posedness and long time behavior of the non-isothermal viscous Cahn-Hilliard equation with dynamic boundary conditions, Dyn. Partial Differ. Equ. 5 (2008), 39-67.

[21] C.G. Gal, Non-local Cahn-Hilliard equations with fractional dynamic boundary, European J. Appl. Math. 28 (2017), 736-788.

[22] G. Gilardi, A. Miranville, G. Schimperna, On the Cahn-Hilliard equation with irregular potentials and dynamic boundary conditions, Commun. Pure Appl. Anal. 8 (2009), 881-912.

[23] G. Gilardi, A. Miranville, G. Schimperna, Long-time behavior of the Cahn-Hilliard equation with irregular potentials and dynamic boundary conditions, Chin. Ann. Math. Ser. B 31 (2010) 679-712.

[24] G. Gilardi, J. Sprekels, Asymptotic limits and optimal control for the Cahn-Hilliard system with convection and dynamic boundary conditions, Nonlinear Anal. 178 (2019), 1-31.

[25] M. Grasselli, H. Petzeltová, G. Schimperna, Asymptotic behavior of a nonisothermal viscous CahnHilliard equation with inertial term, J. Differential Equations 239 (2007), 38-60.

[26] J.-L. Lions, "Quelques Méthodes de Résolution des Problèmes aux Limites non Linéaires", Dunod Gauthier-Villars, Paris, 1969.

[27] A. Lunardi, "Interpolation Theory", third edition, Lecture Notes Scuola Normale Superiore di Pisa, Series Appunti 16, Edizioni della Normale, Pisa, 2018.

[28] A. Miranville, S. Zelik, Robust exponential attractors for Cahn-Hilliard type equations with singular potentials, Math. Methods Appl. Sci. 27 (2004), 545-582.

[29] L. Tartar, Interpolation nonlinéaire et régularité, J. Funct. Anal. 9 (1972), 469-489.

[30] F. Wang, H. Chen, H. Wang, Finite element simulation and efficient algorithm for fractional CahnHilliard equation, J. Comput. Appl. Math. 356 (2019), 248-266.

[31] X.-M. Wang, H. Wu, Long-time behavior for the Hele-Shaw-Cahn-Hilliard system, Asymptot. Anal. 78 (2012), 217-245.

[32] H. Wu, S. Zheng, Convergence to equilibrium for the Cahn-Hilliard equation with dynamic boundary conditions, J. Differential Equations 204 (2004), 511-531.

[33] H. Ye, Q. Liu, M. Zhou, An $L^{\infty}$ bound for solutions of a fractional Cahn-Hilliard equation, Comput. Math. Appl. 79 (2020), 3353-3365.

[34] X. Zhao, C. Liu, On the existence of global attractor for 3D viscous Cahn-Hilliard equation, Acta Appl. Math. 138 (2015), 199-212.

[35] S. Zheng, Asymptotic behavior of solution to the Cahn-Hilliard equation, Appl. Anal. 23 (1986), $165-184$ 
Pierluigi Colli and Gianni Gilardi Dipartimento di Matematica "F. Casorati”

Università di Pavia

Via Ferrata 5

27100 Pavia

Italy

E-mail:pierluigi.colli@unipv.it

Gianni Gilardi

E-mail: gianni.gilardi@unipv.it

Jürgen Sprekels

Department of Mathematics

Humboldt-Universität zu Berlin

Unter den Linden 6

10099 Berlin

Germany

E-mail: sprekels@wias-berlin.de

and

Weierstrass Institute for Applied Analysis and Stochastics

Mohrenstrasse 39

10117 Berlin

Germany

Accepted: 13 April 2021 